NASA Contractor Report 182071

ICASE Report No. 90-47

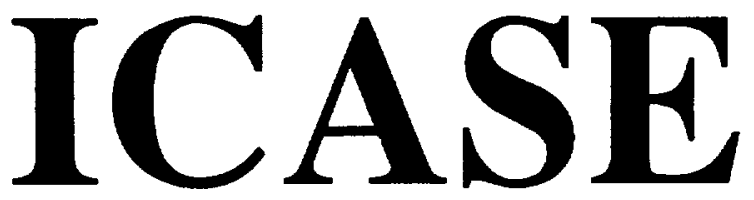

ON THE USE OF LAGRANGIAN VARIABLES IN DESCRIPTIONS OF UNSTEADY BOUNDARY LAYER SEPARATION

Stephen J. Cowley

Leon L. Van Dommelen

Shui T. Lam

Contract No. NAS1-18605

July 1990

Institute for Computer Applications in Science and Engineering NASA Langley Research Center

Hampton, Virginia 23665-5225

Operated by the Universities Space Research Association

เே

$-\pi$

$3 a \frac{a}{a}$

a: 0

$-a_{0}$

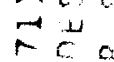

O

$\approx \geq 2$

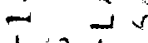

$1 \rightarrow 1$

का

$1+x=$

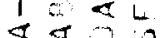

\& 40

$v=c$

$\exists \times 20$ 
-... 


\title{
ON THE USE OF LAGRANGIAN VARIABLES IN DESCRIPTIONS OF UNSTEADY BOUNDARY-LAYER SEPARATION
}

\author{
Stephen J. Cowley ${ }^{1}$ \\ Applied Mathematics 217-50 \\ California Institute of Technology \\ Pasadena, California 91125, USA \\ Leon L. Van Dommelen ${ }^{1}$ \\ Department of Mechanical Engineering \\ FAMU/FSU College of Engineering \\ Tallahassee, Florida 32304, USA \\ Shui T. Lam \\ National Power Technology and Environmental Centre \\ Kelvin Avenue, Leatherhead, Surrey KT22 7SE, UK
}

\begin{abstract}
The Lagrangian description of unsteady boundary-layer separation is reviewed from both analytical and numerical perspectives. We explain in simple terms how particle distortion gives rise to unsteady separation, and why a theory centred on Lagrangian coordinates provides the clearest description of this phenomenon. Included in the review are some of the more recent results for unsteady three-dimensional compressible separation. The different forms of separation that can arise from symmetries are emphasized. Current work includes a possible description of separation when the detaching vorticity layer exits the classical boundary-layer region, but still remains much closer to the surface than a typical bodylengthscale.
\end{abstract}

\footnotetext{
${ }^{1}$ Research was supported by the National Aeronautics and Space Administration under NASA Contract No. NAS1-18605 while the authors were in residence at the Institute for Computer Applications in Science and Engineering (ICASE), NASA Langley Research Center, Hampton, VA 23665.
} 
- 


\section{Introduction}

Mathematical descriptions of fluid flows must yield physical results that are independent of the coordinate system and the choice of mathematical variables. For a given physical model, the choice of mathematical formulation is determined by criteria of simplicity and the insight that the mathematics yields of the underlying physical processes. For many fluid dynamical problems, and particularly for steady flows, the simplest and cleanest mathematics arises from the use of Eulerian coordinates. However, for other flows, such as unsteady flows in which advection dominates diffusion, Lagrangian particle coordinates may be more appropriate.

In this article we show how, over the last decade or so, the use of Lagrangian coordinates has yielded insights into unsteady separation which investigations using Eulerian coordinates in the preceding seventy years had overlooked. By 'separation' we refer to the high-Reynolds-number flow phenomenon by which thin viscous boundary layers generated next to a rigid surface can 'break-away' from that surface. This definition of separation is close to that of both Prandtl (1904) and Sears \& Telionis (1975). In addition it is consistent with J.H.B. Smith's (1989, private communication) alternative, and equivalent, definition that separation occurs when the dominant mode of vorticity transport away from the surface, but within the boundary-layer, is advection.

We note that for two-dimensional flow past a rigid surface, unsteady separation is ordinarily preceded by a stage of flow reversal. However, as Moore (1958), Rott (1956) and Sears (1956) have pointed out: in unsteady problems the occurrence of reversed flow or recirculating eddies need not imply the dramatic 'breakup' of the boundary layer in which vorticity is driven away from the surface by advective forces - the process we call separation. Conversely, the computations of Peridier \& Walker (1989) and the experiments of Didden \& Ho (1985) show separation when the flow in the boundary layer is no longer reversed.

An understanding of separation is of considerable interest in the design of air, land, and sea vehicles because boundary-layer vorticity induces the important transverse 'lift' forces exerted on these vehicles; therefore its ejection has a dramatic impact on the fluid mechanical loads. For similar reasons, separation is important in the flow about obstacles such as chimneys, cooling towers and offshore structures (e.g. oil-rigs), and it plays a role 
in several physiological problems (e.g., the growth of atherosclerotic lesions). Unsteady separation may also be closely related to phenomena that arise in the interior of turbulent boundary layers (e.g., the formation of hairpin vortices). A better grasp of the physical processes involved in separation may help in the construction of improved analytical and computational fluid mechanics methods to describe these flows.

In section 2 we briefly highlight the assumptions inherent in classical boundary-layer theory, and then describe a simple physical model of unsteady separation. At the start of section 3 we review in detail the mathematical description of unsteady, two-dimensional, incompressible separation. This is followed by an outline of the generalisations to compressible three-dimensional flow, including the special cases when there are symmetries present. A theoretical description of a 'weak' form of asymmetric separation, known as 'marginal' separation, is given in section 4 . The differences in marginal separation resulting from imposing symmetry are discussed in section 5 , while closing remarks are given in section 6. In keeping with the theme of this volume we concentrate on the Lagrangian picture of unsteady separation, and refer the reader elsewhere for more comprehensive reviews of steady separation, interacting theories, etc. (e.g. Smith 1986, Ghia 1987, Simpson 1989).

\section{The Classical Boundary-Layer Model}

There are two major approximations involved in deriving the well-known equations of Prandtl's boundary-layer theory. The first is that viscous diffusion can to leading order be ignored except in the direction normal to the local boundary. Usually this is not a severe approximation in the sense that even when classical boundary-layer theory fails, (possibly through the formation of singularities), it is seldom that viscous diffusion parallel to the boundary is the neglected physical effect that needs to be reintroduced. Indeed, although spanwise diffusion is important in Görtler-vortex type flows, there are few instances except fully turbulent flow where streamwise viscous diffusion is dynamically significant; it is this observation that is the rationale behind the parabolised Navier-Stokes equations and other simplified sets of equations such as those proposed by Smith, Papageorgiou \& Elliott (1984) (but see Cowley \& Smith (1985) for a counter-example).

The other major assumption in classical boundary-layer theory is that the thin boundary layer has no significant effect on the pressure distribution close to the wall. Together, 
these two assumptions imply that if some mechanism exists to generate large velocities normal to the boundary there is neither a pressure gradient nor a viscous force induced to oppose the motion; as a result velocities can, in principle, become unbounded (cf. flows governed by the Navier-Stokes or Euler equations, where, except for special cases, pressure gradients, etc., are generated to inhibit the development of large velocities).

In the case of unsteady separation, such unbounded velocities are in fact predicted by classical boundary-layer solutions. These velocities are self-induced. To see how this can occur, consider a little mass of incompressible fluid, (a fluid 'particle'), inside a twodimensional boundary layer. If the fluid particle begins to shorten considerably in the direction parallel to the boundary, then to conserve volume, the particle must simultaneously expand in the orthogonal direction (see the schematic in figure $1 a$ ). Within the classical boundary-layer model there is no resistance to such a distortion, and thus it is possible to squash a fluid particle to 'zero-thickness' in the direction parallel to the boundary, and consequently to 'infinite' thickness in the direction normal to the boundary. As a result an 'infinite' velocity is generated normal to the boundary, and the fluid particles above the squashed one are ejected from the boundary layer.

The central role of the deformed fluid particle in the above physical description suggests that a mathematical analysis of unsteady boundary-layer separation based on Lagrangian coordinates may be significantly simpler than an Eulerian one. Further, the observation that viscous diffusion normal to the boundary is seldom important also suggests that the new physics which needs to be introduced into the classical boundary-layer model once the fluid particle has been excessively deformed, is a variation in pressure gradient normal to the boundary. Indeed, starting from the Navier-Stokes equations it is possible to formulate so-called asymptotic 'interaction' problems which account for the pressure disturbances induced by the rapid stretching of the fluid particle (Elliott, Cowley \& Smith 1983). As demonstrated in section 4, Lagrangian coordinates are also advantageous in solving a special case when the interaction is in some sense 'weak'.

\section{Unsteady Boundary-Layer Separation}

\subsection{Lagrangian Formulation}

Initially we will focus on two-dimensional incompressible flows as described by Van 
Dommelen \& Shen (1982), and then we will indicate how the analysis was generalized to three-dimensional compressible flows by Van Dommelen \& Cowley (1990) (henceforth referred to as VDC). For definiteness we assume that fluid with Newtonian viscosity $\nu$ and density $\rho$ is flowing with a typical velocity $U$ past a body of typical dimension $D$. Lengths, velocities, time and pressure are then non-dimensionalised by $D, U, D / U$ and $\rho U^{2}$ respectively, and a Reynolds number

$$
R=U D / \nu,
$$

which we assume to be large, is introduced. As is conventional in boundary-layer theory, coordinates $\left(x, R^{-\frac{1}{2}} y\right)$ are taken parallel and normal, respectively, to the surface of the body, while the corresponding velocity components are $\left(u, R^{-\frac{1}{2}} v\right)$. The two-dimensional boundary-layer equations in Eulerian coordinates are then (e.g., Rosenhead 1963)

$$
\begin{gathered}
u_{t}+u u_{x}+v u_{y}=-p_{x}+u_{v y} \\
p_{y}=0, \quad u_{x}+v_{y}=0 .
\end{gathered}
$$

If the surface of the body is fixed and impermeable, the boundary conditions on the surface are

$$
u=v=0 \quad \text { on } \quad y=0
$$

Far from the surface, the boundary-layer solution must match with a known inviscid flow solution that provides a slip-velocity $u_{\varepsilon}(x, t)$ on the surface of the body, i.e.

$$
u \rightarrow u_{e} \quad \text { as } \quad y \rightarrow \infty
$$

Using this matching condition it follows that the pressure gradient is a known function of $x$ and $t$, i.e.,

$$
-p_{x}=u_{e t}+u_{\varepsilon} u_{e x}
$$

Now we will formulate the same problem in Lagrangian coordinates, using fluid particles as the basis of the coordinate system. A convenient coordinate system for these particles, $(\xi, \eta)$, is given by their Eulerian position at some chosen instant, say $t=t_{l}$; i.e.,

$$
\boldsymbol{\xi} \equiv(\xi, \eta)=(x, y) \quad \text { at } \quad t=t_{l}
$$


The velocity components of the flow are then related to the fluxions of position by the kinematic relations

$$
u(\boldsymbol{\xi}, t)=\dot{x}(\boldsymbol{\xi}, t), \quad v(\boldsymbol{\xi}, t)=\dot{y}(\boldsymbol{\xi}, t),
$$

where a dot represents a Lagrangian time derivative.

In Lagrangian coordinates, conservation of mass (3.1c) can be expressed in terms of a conserved Jacobian determinant:

$$
J(x, y) \equiv\left|\begin{array}{ll}
x_{, \xi} & x_{, \eta} \\
y, \xi & y, \eta
\end{array}\right|=1
$$

where a subscript comma denotes a Lagrangian derivative. In these coordinates the momentum equation $(3.1 a)$ becomes

$$
\dot{u}=-p_{x}+D_{y}\left(D_{y} u\right)
$$

where from (3.2c) the pressure gradient is a known function of $x$ and $t$, and $D_{y}$ is the Eulerian $y$-derivative. The latter can be written in the Lagrangian form (e.g., Shen 1978a):

$$
D_{y} u=J(x, u)=x_{, \xi} u, \eta-x_{, \eta} u_{, \xi}
$$

In a Lagrangian formulation the boundary conditions $(3.2 a, b)$ remain essentially unchanged, although they need to be supplemented by conditions on $(x, y)$ :

$$
\begin{gathered}
(x=\xi, u=0) \text { and }(y=0, v=0) \text { on } \eta=0, \\
\dot{x}=u \rightarrow u_{e}(x, t) \text { as } \eta \rightarrow \infty .
\end{gathered}
$$

The main simplification of a Lagrangian approach is evident from the system of equations $(3.4 a, 6 a, 6 b, 7 a, 7 c)$ that provides sufficient information to solve for the position $x$ and velocity $u$ parallel to the surface independently of the position $y$ and velocity $v$ normal to the surface. It is this decoupling that is the key to much of the analysis that follows. Once $x$ is known, the normal particle position $y$ can be found by integration of the Jacobian (3.5) along lines of constant $x$; in particular

$$
y=\int_{0}^{s} \frac{d s}{\sqrt{x_{, \xi}^{2}+x_{, \eta}^{2}}},
$$


where $d s^{2}=d \xi^{2}+d \eta^{2}$, and the integral is performed in the Lagrangian $(\xi, t)$ coordinate system along the lines of constant $x$ and $t$, i.e., lines that in physical space are vertical through the boundary layer.

\subsection{Hypothesis}

To make further analytical progress it is necessary to make an assumption; namely that up to and including the time of separation, the solution for the projected position $x$ remains a regular function of $\xi$ and $t$. Any singularities that develop will then be associated with irregularities in the continuity equation. From either (3.5) or (3.8) such singularities can occur at a fluid particle, say $\xi=\xi_{1}$, if at some time, say $t=t_{\infty}$, a stationary point develops in $x(\xi, t)$ (Van Dommelen \& Shen, 1980), i.e. if

$$
x_{, \xi}=x_{, \eta}=0 \quad \text { at } \quad \boldsymbol{\xi}=\boldsymbol{\xi}, t=t_{\triangleleft} .
$$

There are several arguments in favor of the regularity of $x$, and the implication that singularities form only in $y$. First, if $x$ is assumed to be regular, then the analytic structure of several separation processes previously studied using Eulerian coordinates can be recovered by a simpler Lagrangian analysis (VDC). Second, Van Dommelen (1981) showed analytically that the inviscid version of $(3.4 a, 6 a, 7 c)$ has solutions that are regular functions of the Lagrangian variables; he also showed that if (3.9) was satisfied $y(\xi, t)$ and $v(\xi, t)$ become singular. Although this analysis can be extended by expanding in powers of a small coefficient of viscosity, the example is somewhat artificial because during most of the evolution of the boundary layer, viscous effects are significant and cannot be neglected. Similar analyses demonstrating the analyticity of the projected position for the case of a three-dimensional inviscid flow with a symmetry line have been presented by Van Dommelen (1981) for the flow on the symmetry line, and Stern \& Paldor (1983), Russell \& Landahl (1984), and Stuart $(1988,1989)$ for the flow near the symmetry line (see also VDC).

However, the most convincing argument in favor of the assumption of regularity comes from numerical solutions of the Lagrangian boundary-layer equations. For example, Van Dommelen \& Shen's (1980) computation of the boundary layer on an impulsively started circular cylinder provided direct numerical evidence on règularity of solutions $x(\xi, t)$ to the momentum equation. Further, the Lagrangian computations are in remarkably close 
agreement with the results obtained in terms of Eulerian coordinates by Cowley (1983) using a series extension technique, by Ingham (1984) using a spectral method, by Matsushita, Murata \& Akamatsu (1984) using an integral method, and by Henkes \& Veldman (1987), Riley \& Vasantha (1989a) and Puppo (1990). using finite-difference schemes. The fact that all these different formulations and methods of solution produce results in excellent agreement with one another until very close to the breakdown of the solution at separation, strongly suggests that the regularity hypothesis in Lagrangian coordinates is correct at least for the particular example of the impulsively started cylinder $\dagger$.

Further support for the regularity of solutions of the classical boundary-layer equations in Lagrangian coordinates is given by many other Lagrangian numerical calculations, e.g.: (a) flow over a translating and rotating cylinder (Shen \& Wu, 1988), (b) starting flow over an airfoil (Wu, 1989), (c) flow on rotating and translating spheres (Van Dommelen 1987, 1990), (d) impulsively started flow through a curved pipe (Lam, 1988), and (e) vortexinduced boundary-layer flow (Peridier \& Walker, 1989). In particular, Van Dommelen (1990) has performed high-resolution numerical calculations in a study of the boundary layer at the equatorial plane of a spinning sphere - he found no evidence of singular behavior in the solution of the momentum equation up to and including the start of separation.

Numerical calculations cannot, however, prove that solutions to the momentum equation are regular prior to separation. Besides, such a proof may be complicated because (i) after a stationary point has developed the solution to the momentum equation can become singular (Van Dommelen, 1990), and (ii) at large times the solution can become exponentially close to a singularity (see section 5 ). In absence of a proof, for the rest of this article we will assume that the solution for $x$ is indeed regular.

\section{3 Moore-Rott-Sears Conditions}

As indicated above, the assumption that $x(\xi, t)$ is analytic implies that singularities can develop only in the continuity equation, and only at times at which the Lagrangian

\footnotetext{
†t should be noted that some of the earlier Eulerian, finite-difference computations gave different results, e.g., those of Telionis \& Tsahalis (1974), Wang (1979), and Cebeci (1986). We believe that these inconsistencies are not independently supported and that they do not show that the regularity hypothesis is invalid.
} 
derivatives (3.9) vanish. This condition implies that for all infinitesimal changes in fluid particle, $\partial \boldsymbol{\xi}$, the corresponding change in particle position is

$$
\partial x=\partial \xi \cdot \nabla_{\xi} x=0
$$

Physically this means that an infinitesimal particle volume $\partial \xi \partial \eta$ around point $s$ has been squashed to zero size in the $x$-direction parallel to the wall. Because particle volume is conserved, the compression in this direction is compensated for by a rapid expansion in the $y$-direction. This drives the fluid above the squashed region $\partial \xi \partial \eta$ 'far' from the wall to form a separating vorticity layer (cf. the physical description given in section 2). Such a process constitutes separation in the sense of Sears \& Telionis (1975), since the particle distance from the wall becomes too large, 'infinite', to be described by the usual boundary-layer scale.

Yet a Lagrangian approach does more than just provide this natural physical description: it also makes it simple to verify that two properties known as the Moore-Rott-Sears conditions are satisfied at separation (Sears \& Telionis 1975). In the present context the first of these conditions asserts that the separation structure moves along the wall with the velocity of the squashed fluid particle. Therefore in a system that moves with the separation structure, the velocity profile will be zero at the squashed particle. The second condition is that the squashed particle has zero vorticity, which implies that the velocity profile also has a stationary point at the particle.

We will be most concerned with the onset of separation and the particle which is the first to be squashed to zero size in the streamwise direction (henceforth $\xi$, will denote this particle and $t$, will denote the time of the onset of separation). That the first MRS condition is satisfied at $t$, follows immediately from the asymptotic scaling $(3.15 \mathrm{c})$ derived in the next subsection. However, solutions to the momentum equation can be found at later times, even if their physical relevance is questionable (Elliott et al., 1983). For these later times the first MRS condition follows directly from the requirement, (3.9), that the Lagrangian derivatives vanish:

$$
\frac{d}{d t} x\left(\boldsymbol{\xi}_{\mathrm{MRS}}, t\right)=\dot{x}+\frac{d \boldsymbol{\xi}_{\mathrm{MRS}}}{d t} \cdot \nabla_{\boldsymbol{\xi}} x=\dot{x}\left(\boldsymbol{\xi}_{\mathrm{MRS}}, t\right)
$$

where $\xi_{\mathrm{MRS}}(t)$ indicates the Lagrangian coordinate of a stationary point. 
The second MRS condition of zero vorticity is a consequence of $(3.6 b, 9)$ because

$$
D_{\nu} u=0 \text { when } \nabla_{\xi^{x}}=0 .
$$

Experimental confirmation of the MRS conditions in unsteady flow is given by Didden \& Ho (1985). For verification of these conditions in other unsteady classical boundary-layer solutions, see, e.g., Williams (1977), and Van Dommelen \& Shen (1983a).

\subsection{Asymptotic Description}

The regularity hypothesis, and the simplicity of the condition of vanishing Lagrangian derivatives (3.9), enabled Van Dommelen \& Shen (1982) to obtain a more precise description of separation near the squashed particle $\xi$, at times close to the initial separation time $t_{s}$. To do this they formed a local Taylor series expansion for the regular solution to the momentum equation near the stationary point $\left(\boldsymbol{\varepsilon}_{s}, t_{s}\right)$, and then expanded the singular solution of the continuity equation in an asymptotic series.

To be more specific, if the function $x(\xi, t)$ is a regular function of $\xi$ and $t$, then close to $\xi_{\Perp}, t_{t}$, it can be expanded as

$$
\begin{gathered}
x=x_{\triangleleft}+\sum_{i, j} \frac{1}{2} \delta \xi_{i} \delta \xi_{j}\left(x_{, i j}\right),+\sum_{i, j, k} \frac{1}{6} \delta \xi_{i} \delta \xi_{j} \delta \xi_{k}\left(x_{, i j k}\right)_{\mathbf{s}}+\ldots \\
+\delta t\left(\dot{x}_{\mathbf{d}}+\sum_{i} \delta \xi_{i}\left(\dot{x}_{, i}\right)_{\mathbf{\imath}}+\ldots\right)+\ldots,
\end{gathered}
$$

where $\left(\xi_{1}, \xi_{2}\right)=(\xi, \eta), x_{, j}=x_{, \xi_{j}}, \delta \xi_{j}=\xi_{j}-\xi_{j,}, \delta t=t-t_{\mathrm{s}}$, and the stationary point condition (3.9) has been used. This expression can be simplified by the transformation

$$
l_{i}=\sum_{j} a_{i j} \delta \xi_{j}
$$

which shifts the Lagrangian coordinate system to the separation particle, $s$, and with a suitable choice of $a_{i j}$, rotates it so as to eliminate the mixed partial derivative $(x, 12)$, in the new coordinate system. Henceforth we will adopt the convention of omitting the subscripts comma and $s$ if they occur together, i.e. $x_{i}=\left(x_{, i}\right)$. The Taylor series expansion $(3.13 a)$ becomes

$$
x=x_{\boldsymbol{\imath}}+\sum_{i} \frac{1}{2} x_{i i} l_{i}^{2}+\sum_{i, j, k} \frac{1}{6} x_{i j k} l_{i} l_{j} l_{k}+\ldots+\delta t\left(\dot{x}_{\bullet}+\sum_{i} \dot{x}_{i} l_{i}+\ldots\right)+\ldots
$$


where the derivatives are with respect to the new coordinate system.

If $t$, is the first time that a stationary point occurs, the Taylor series coefficients in the rotated coordinate system cannot be completely arbitrary because the singularity condition may not be satisfied anywhere for $\delta t<0$. On expanding the condition (3.9) also in a Taylor series it is readily verified that one of the coefficients $x_{11}$ and $x_{22}$ must be zero if $\delta t=0$ is the first time that a singularity forms; for definiteness $\left(l_{1}, l_{2}\right)$ are reordered such that $x_{11}$ vanishes. The Taylor series $(3.13 c)$ can now be reduced to

$$
x \sim x(\xi, t)+\frac{1}{2} x_{22} l_{2}^{2}+\frac{1}{6} x_{111} l_{1}^{3}+\ldots+\delta t\left(\dot{x}_{1} l_{1}+\ldots\right)+\ldots,
$$

where only those terms which will turn out to be important at leading order have been displayed.

VDC discuss flows where some of the coefficients in (3.13d) are zero due to symmetries that impose additional constraints (also see below). For this part of the analysis it is assumed that the values of these derivatives can be completely arbitrary and will in general be nonzero; $c f$. the values given by Van Dommelen (1981) for the circular cylinder. There are, however, the following constraints on the signs:

$$
x_{22} \dot{x}_{1}<0 \quad, \quad x_{111} \dot{x}_{1}<0 \quad
$$

The first of these simply fixes the positive $l_{1}$-direction, but the second is required if the expression $(3.13 d)$ is to be free of stationary points for $\delta t<0$.

Under the above conditions, at times close to separation the lines of constant $x$ in the Lagrangian domain appear as sketched in figure $2 a$. At the separation time, the fold at the separation particle $s$ collapses to a cusp. Note that physically these lines are simply vertical straight lines through the boundary layer, as indicated in the inset of figure $2 a$.

Next we turn to the integration of the Jacobian (3.5) to find the $y$-position of the particles. This Jacobian is preserved by the transformation to the new local Lagrangian coordinates, and has characteristics

$$
\frac{d l_{1}}{d y}=-x_{22} l_{2}+\ldots \quad, \quad \frac{d l_{2}}{d y}=\frac{1}{2} x_{111} l_{1}^{2}+\dot{x}_{1} \delta t+\ldots
$$

A singularity occurs when both right hand side expressions vanish. Near the point $s$, the first right hand side is zero on a surface approximating the $l_{2}=0$ plane, while $(3.13 f$ ) insures that the second right hand side does not vanish in that plane when $\delta t<0$. 
At $t=t$, the boundary-layer approximation is obviously no longer valid because $y$ becomes infinite at the stationary point (see (3.8)). However, at times shortly before $t$. a local description of the flow field can be obtained by asymptotic expansions. Following the guiding principles of Van Dyke (1975), the aim is to scale the Lagrangian coordinates $l_{i}$ and the position coordinates $x$ and $y$ to variables $L_{i}, X$, and $Y$ such that in the 'inner' asymptotic region the characteristic equations (3.14) are non-singular. This suggests that the $\delta t$ term in (3.14b), which ensures the absence of singular points for $\delta t<0$, should be retained. Further, for $\delta t=0$ we want to match the solution close to the stationary particle to a solution for $y$ which is regular away from this point. Thus those terms that ensure the absence of singular points away from particle $\xi_{\text {a }}$ at time $\delta t=0$, i.e., the $l_{1}^{2}$ and $l_{2}$ terms in (3.14), must also be retained. The appropriate scalings are therefore

$$
\begin{gathered}
l_{1}=|\delta t|^{\frac{3}{2}} L_{1} \quad, \quad l_{2}=|\delta t|^{\frac{3}{4}} L_{2}, \\
\bar{x}=x-x\left(\xi_{\bullet}, t\right)=|\delta t|^{\frac{3}{2}} X \quad, \quad y=|\delta t|^{-\frac{1}{4}} Y .
\end{gathered}
$$

These scalings suggest that the separation process occurs in a relatively thin strip of size $|\delta t|^{3 / 2}$ moving with a velocity $\dot{x}\left(\xi_{\imath}, t\right)$.

For the scaling (3.15), the analytic solution for $Y$ can be found by integration of (3.14). The result is:

$$
Y \sim \int_{-\infty}^{L_{0}} \frac{d L}{\sqrt{P(L ; X)}} \pm \int_{L_{1}}^{L_{0}} \frac{d L}{\sqrt{P(L ; X)}}
$$

where

$$
P(L ; X)=-\frac{1}{3} x_{22}\left(x_{111} L^{3}-6 \dot{x}_{1} L-6 X\right),
$$

and $L_{0}(X)$ is the real root of the cubic $P$. This root is a unique and continuous function of $X$ since $P$ is a monotonically decreasing function of $L$ from $(3.13 e, f)$.

The dependence on the coefficients of the Taylor series can be scaled out by the transformations (Van Dommelen 1981):

$$
L_{1}=\left(-\frac{2 \dot{x}_{1}}{x_{111}}\right)^{\frac{2}{2}} L_{1}^{*}, \quad X=-\frac{2}{3} \dot{x}_{1}\left(-\frac{2 \dot{x}_{1}}{x_{111}}\right)^{\frac{1}{2}} X^{*}, \quad Y=\left(-\frac{9}{2 \dot{x}_{1} x_{111} x_{22}^{2}}\right)^{\frac{1}{4}} Y^{*} .
$$

In terms of the new variables (3.16) reduces to

$$
Y^{*}\left(L_{1}^{*}, X^{*}\right) \sim \frac{2}{\Lambda} F\left(\frac{\pi}{2} \mid m\right) \pm \frac{1}{\Lambda} F(\varphi \mid m)
$$


where

$$
\begin{gathered}
\Lambda\left(X^{*}\right)=\left(3\left(L_{0}^{*}+1\right)\right)^{\frac{1}{4}}, \quad \varphi\left(L_{1}^{*}, X^{*}\right)=2 \arctan \left(\frac{\sqrt{L_{0}^{*}-L_{1}^{*}}}{\Lambda}\right) \\
m\left(X^{*}\right)=\frac{1}{2}+\frac{3 L_{0}^{*}}{4 \Lambda^{2}}, \quad L_{0}^{*}\left(X^{*}\right)=\left(X^{*}+\left(1+X^{*}\right)^{\frac{1}{2}}\right)^{\frac{2}{3}}+\left(X^{*}-\left(1+X^{*}\right)^{\frac{2}{2}}\right)^{\frac{1}{3}}
\end{gathered}
$$

and $F(\phi \mid m)$ is the incomplete elliptic integral of the first kind. Further terms in the asymptotic expansion can be found in principle.

The choice of sign of the square-roots and the limits of integration, etc. in $(3.16 a, 18 a)$ are determined by the topology of the lines of constant $X$, as shown in figure $2 a$. These lines of can be divided into three segments corresponding to three asymptotic regions. This subdivision is schematically shown by the variation in line thickness in figure $2 a$. The lower segments start at the wall and extend upward towards the vicinity of the separation particle. Because the Jacobian is nowhere singular along these segments, the $y$-positions of the fluid particles remain finite on the boundary-layer scale, i.e., the scaled coordinate $Y$ is small. Therefore, these lower segments yield a layer of particles at the wall with a thickness comparable to that of the original boundary layer; this is shown schematically in figure $1 b$.

Along the central segments, the lines of constant $X$ pass through the vicinity of the separation particle. Here the $y$-position of the particles grows rapidly, and is given in scaled form by (3.18). Thus the central segments cause the intermediate, thicker, layer illustrated in figure $1 b$. The topology of the central segments in the Lagrangian domain, figure $2 a$, determines the choice of sign in $(3.16 a, 18 a)$. From $(3.13 e, f, 14 a, b)$ it follows that on integrating upwards, $L_{1}$ increases from large negative values towards $L_{0}(X)$. Since $Y$ increases, along this part the negative sign in (3.16a) applies. At position $L_{0}$, the lines of constant $X$ turn around in the Lagrangian domain and $L_{1}$ again tends to $-\infty$; along this second part the positive sign in (3.16a) applies.

Along the third segments, the lines of constant $X$ proceed upwards toward the external flow. As in the lower segments, the Jacobian is no longer small here. Thus the changes in $y$ are finite on boundary-layer scale, and the third segments cause a layer of particles with a boundary-layer scale thickness, atop the central region (see figure $1 b$ ). 
Taking the boundary-layer scaling of the normal position into account, it follows that the separation structure is one in which the boundary layer divides into a central layer of physical thickness proportional to $R e^{-\frac{1}{2}}|\delta t|^{-\frac{1}{4}}$, between two 'sandwich' layers of thickness proportional to $R e^{-\frac{1}{2}}$.

\subsection{Interpretation}

We now turn to the physical interpretation of these results. The boundary-layer thickness is asymptotically determined by the position of the upper particle layer in figure $1 b$; letting $L_{1}^{*} \rightarrow-\infty$ along the positive branch of $(3.18 a)$, we obtain from $(3.16,18)$ the scaled thickness of the expanding central region near separation as

$$
Y^{+^{*}}\left(X^{*}\right)=2 \int_{-\infty}^{L_{0}^{*}} \frac{d L^{*}}{\sqrt{2 X^{*}-L^{*}-3 L^{*}}}=\frac{4}{\Lambda} F\left(\frac{\pi}{2} \mid m\right) .
$$

The function $Y^{+^{*}}\left(X^{*}\right)$ gives the general shape of the boundary-layer thickness, and is illustrated in figure $2 b$. Figure $2 c$ is a plot of boundary-layer displacement thickness at different times for an impulsively started circular cylinder calculation with $u_{\varepsilon}=\sin (x)$ for $t>0$ in (3.2b) (Van Dommelen 1981). In agreement with the scaling (3.15d), the numerical calculation suggests that the displacement thickness becomes infinite at separation; further the two graphs are in qualitative agreement regarding the shape of displacement thickness near the separation particle. While a quantitative comparison is not possible in this case due to the difficulty of obtaining accurate numerical solutions when $|\delta t|^{\frac{1}{4}}$ is small, confirmation of the scaling (3.15d) is given by Peridier \& Walker (1989) in their Lagrangian calculations of vortex-induced separation. They assume that the maximum displacement thickness is proportional to $|\delta t|^{-M}$, and show that $M=0.253 \pm 0.003$.

The particle propagation velocity $\dot{\bar{x}}$ that causes the accumulation of particles at the separation line is given to leading order by (see $(3.13,15)$ )

$$
\dot{\bar{x}}=\dot{x}-\dot{x}\left(\xi_{\bullet}, t\right) \sim|\delta t|^{\frac{1}{2}} \dot{x}_{1} L_{1} . .
$$

To describe this in the more familiar Eulerian coordinates, the transcendental relationship (3.18) must be inverted. The inversion has been performed numerically. Contours of $L_{1}^{*}$, or equivalently contours of $\dot{\bar{x}}$ or $\dot{x}$, in the $\left(X^{*}, Y^{*}\right)$ plane are illustrated in figure $2 d$. The topology of this figure for $|\delta t| \approx 0$ is close to the computed lines of constant velocity presented by Van Dommelen (1981) for finite $|\delta t|$ - reproduced here as figure $2 e$. 
Another point of interest is the shape of the velocity profiles. According to (3.20), as separation is approached the Eulerian velocity profiles develop a large flat region of nearly constant velocity near a local maximum or minimum - see figure $2 f$. Confirmation of both the flat, almost constant, region and the turning point for the impulsively moved cylinder problem is given by Van Dommelen \& Shen (1980). Peridier \& Walker (1989) also find an almost constant region in their velocity profiles for vortex-induced separation, although, rather intriguingly, there is no clear turning point; indeed, their profiles seem closer to an inflection point at $\xi=\xi_{a}, t=t_{s}$.

The shapes of the velocity profiles in the sandwich layers at the edges of figure $2 f$ cannot be found from asymptotic analysis because they depend on the precise details of the earlier evolution (see the remarks below (3.21)). It should also be noted that while there is a local minimum (or maximum) in the velocity profile at separation, the existence of such a turning point is not necessarily an indication that separation is about to occur. For example, for the impulsively started circular cylinder, a minimum in the velocity profiles develops quickly, after $\frac{1}{6}$ diameter motion, yet separation occurs much later, after $\frac{3}{4}$ diameter motion (Van Dommelen \& Shen, 1980).

A more useful indication of the start of separation is the rapid transverse expansion of the lines of constant vorticity near the turning point in velocity. This occurs because the above analysis is inviscid to leading order, so that the vorticity lines follow the motion of the boundary-layer particles. The corresponding asymptotic topology of contours of $\partial L_{1}^{*} / \partial Y^{*}$ is shown in figure $2 g$, which is close to the computed vorticity lines presented by Van Dommelen (1981) for a time near separation (see figure $2 h$ ).

So far, the analysis has concentrated on the structure of the boundary layer in the rapidly expanding central region. The asymptotic structures of the upper and lower sandwich layers still need consideration. The displacement of the upper sandwich layer by the central region is given by (3.19) in scaled form, and it is convenient to use the Prandtl transformation to account for it as a shifted transverse position coordinate:

$$
\tilde{y}=y-y^{+}(x, t)
$$

where to leading order

$$
y^{+}(x, t) \sim|\delta t|^{-\frac{1}{4}} Y^{+}(X)
$$


The structures of both layers then take the form of regular Taylor expansions. In terms of Eulerian coordinates, they are:

$$
(\dot{x}, \dot{y})=\sum_{m r \geq 0} x^{m} \delta t^{r}\left(u_{m r}^{-}(y), v_{m r}^{-}(y)\right) \quad \text { and }(\dot{x}, \dot{y})=\sum_{m r \geq 0} x^{m} \delta t^{r}\left(u_{m r}^{+}(\tilde{y}), v_{m r}^{+}(\tilde{y}),\right),
$$

respectively, where the sums run over the non-negative integers. The $u_{m+}^{ \pm},(m, \geq 0, r \geq 1)$ and the $v_{m+}^{ \pm},(m, r \geq 0)$ are determined in terms of the $u_{m 0}^{ \pm}$, but the latter functions are indeterminate due to the dependence of the solution on earlier times (Van Dommelen 1981). The $u_{m 0}^{ \pm}$must, however, satisfy the boundary conditions (3.2a) at the wall, and match both at the outer edge of the boundary layer (see (3.2b)) and with the central inviscid low-vorticity region (see Van Dommelen (1981) for the precise conditions). Similarly, the solutions in the two sandwich layers and the central layer can be shown to match with a boundary layer of standard width as $|X| \rightarrow \infty$.

\subsection{Subsequent Stages}

Naturally, the singularity structure derived here will not remain asymptotically correct arbitrarily close to the time of actual singularity $t=t_{s}$, because the normal velocity above the central inviscid region becomes infinite when the singularity forms. To be more specific, at times close to $t$, the boundary layer thickens to $O\left(R^{-\frac{1}{2}}|\delta t|^{-\frac{1}{4}}\right)$ in a region with a streamwise extent $O\left(|\delta t|^{\frac{3}{2}}\right)$. In moving past this locally thickened region of the boundary layer, the fluid above the boundary layer experiences a viscous displacement velocity of $O\left(R^{-\frac{1}{2}}|\delta t|^{-\frac{7}{6}}\right)$. Just above the boundary layer, there is an asymptotic region, 'an upper deck', where this velocity perturbation is reduced to zero as a result of an induced normal pressure gradient. The perturbation in this region is irrotational, and hence the induced pressure-gradient perturbation is found from Bernoulli as

$$
\Delta p_{x}=O\left(R^{-\frac{1}{2}}|\delta t|^{-\frac{13}{4}}\right)
$$

When this induced pressure gradient is as large as the $\bar{x}_{t t}$-accelerative forces in the expanding central region, i.e. from (3.15c)

$$
\Delta p_{x}=O\left(|\delta t|^{-\frac{1}{2}}\right)
$$

there is a 'triple-deck' interaction. This occurs when

$$
\delta t=O\left(R e^{-\frac{2}{12}}\right)
$$


at which time the scaled boundary-layer displacement thickness has increased to $O\left(\operatorname{Re}^{\frac{1}{22}}\right)$ (Elliott et al. 1983).

Confirmation of the scaling (3.22c) from accurate solutions of the Navier-Stokes equations is not yet available. However, Peridier \& Walker (1989) have presented solutions of the interactive boundary-layer equations for a range of large Reynolds numbers. They find that a singularity forms even with the interactive effect included, say at $t_{s}(R)$. On assuming that the difference in singularity times is proportional to $R^{-M}$, they find from a regression analysis that $M=0.190 \pm 0.024$. Since $\frac{2}{11}=0.182$, this suggests that the singularity time is shifted by an amount consistent with the scaling $(3.22 c)$.

Their results also show that at $R=10^{5}$ and $R=10^{8}$ the singularity time is changed by $35 \%$ and $10 \%$ respectively. This suggests that the relatively small power of the Reynolds number in $(3.22 c)$ can lead to large differences between theory and experiment at moderate Reynolds numbers. For instance, the numerical calculation of Van Dommelen \& Shen (1980) for the impulsively started cylinder predicts separation for infinite Reynolds number at a position $111^{\circ}$ from the front stagnation point, at a time $t_{c}(\infty)=0.75 D / U$ where $U$ and $D$ are the velocity and diameter of the cylinder. On the other hand, experiments (e.g. Bouard \& Coutanceau 1980, Nagata, Minami \& Murata 1979, Nagata, Funada, Kawai \& Matsui 1985) and numerical solutions of the Navier-Stokes equations (e.g. Collins \& Dennis 1973, Ta Phuoc Loc \& Bouard 1985, Pepin 1990) at Reynolds numbers less than $10^{5}$, suggest that the boundary layer breaks away from the surface at significantly greater angles and at later times; although it is of course difficult at finite Reynolds numbers to say exactly when separation has occurred. However, if we hypothesize that the experimentally observed secondary vortex forms within an asymptotically short time of $t_{\mathbb{s}}(\infty)$ (as yet there is no firm supporting analysis for this assumption), then the trend is towards the theoretically predicted results as the Reynolds number increases; in particular the time, $t_{v}$, at which the secondary vortex forms decreases towards $t_{\mathrm{e}}(\infty): U t_{\mathrm{v}} / D=1.49,1.00,0.94$, for $R=550,3000,9500$ respectively (Pepin 1990, private communication). More detailed numerical calculations for $R>10^{5}$ are needed to determine how large the Reynolds number needs to be for the separation time to be within, say, $5 \%$ of its asymptotic value.

Another problem for which accurate numerical solutions are still required, is the description of the flow on the $\delta t=O\left(R e^{-\frac{2}{13}}\right)$ timescale (see Elliott et al. (1983) for a formu- 
lation). One of the main numerical difficulties in this problem arises from the unbounded matching conditions at the edge of the central inviscid region. Rather than trying a direct attack, in the next section we will address a considerably more attractive interactive problem for a case in which the separation is 'weak'.

Also, in lieu of a solution for $\delta t=O\left(R e^{-\frac{2}{12}}\right)$, we note that a number of related interactive problems have been studied. For instance, Cowley, Duck \& Tutty (1988) (see also Cowley \& Van Dommelen, 1990) have obtained numerical solutions to the viscous triple-deck equations which are in line with the suggestion of Tutty \& Cowley (1986) that solutions to these equations might terminate in a finite-time singularity. In particular, for a growing Tollmien-Schlichting wave they find that while the pressure remains a continuous function, its spatial derivative, and the wall shear, become unbounded at a finite time. Their asymptotic analysis, and the simultaneous work of Smith (1988), is in broad agreement with the numerical results.

Further, as mentioned above, Peridier \& Walker (1989) have performed interactive boundary-layer calculations using Lagrangian coordinates. They find singularities with the same qualitative form as Cowley et al. (1988), and show that if the maximum wall shear is assumed proportional to $\left(t_{0}(R)-t\right)^{-M}$, then a regression analysis yields $M=0.252 \pm 0.016$ - this is consistent with one of the singularities presented by Smith (1988).

All these results suggest that while a 'triple-deck' type interaction modifies the form of the classical boundary-layer singularity, it is not sufficient to eliminate finite-time singularities from the interactive equations. In an attempt to understand what happens once the second singularity has developed (i.e. assuming one forms on the $R^{-\frac{2}{11}}$ timescale), Smith (1990) has proposed an analysis for an even shorter asymptotic timescale.

As yet no asymptotic solutions have been presented which yield a description of the flow for finite times beyond $t$, (but see Sychev (1979), Van Dommelen \& Shen (1983b), E1liott et al (1983) for interactive models of upstream slipping separation points). However, we note that accurate Lagrangian solutions of the classical boundary-layer momentum equations can be found for finite times after separation without difficulty (e.g., Van Dommelen 1990); the question then arises whether these solutions have any relevance at all. At times beyond separation, vertical lines through the boundary layer appear in Lagrangian space as shown in figure $2 i$ rather than figure $2 a$ (see VDC for similar figures when the 
separation is symmetric). Although $y$ is indeterminate for the shaded particles, the continuity equation can still be integrated along all lines of constant $x$ that start at the wall; a singularity develops only on the line passing through the saddle point in figure $2 i$. However, the physical relevance of such solutions is doubtful - especially for all those lines of constant $x$ that contain particles that have at some time been at a position, $x_{M \mathrm{R} s}$, where the stationary point condition (3.9) was satisfied. These lines correspond to a growing band towards the right of the line through the saddle point in figure $2 i$. Additional restrictions would exist if the interaction region accelerates particles to high streamwise velocity or induces an appreciable pressure perturbation at finite distances. Yet such effects would have to preserve energy and the center of vorticity (Van Dommelen 1981).

\subsection{Three-Dimensional Compressible Flow}

The analysis described so far generalizes to the case of compressible, three-dimensional flow through the inclusion of an energy equation and a second momentum equation in the $z$ direction along the wall. Both these equations are also independent of the normal position $y$ of the particles (Shen $1978 \mathrm{~b}, \mathrm{VDC}$ ). The regularity hypothesis is now that the projected surface positions $x$ and $z$, plus the density $\rho$, are regular functions of the Lagrangian coordinates $\xi=(\xi, \eta, \zeta)$ and time $t$ (it is assumed that no shocks are present at the point where separation starts).

The continuity equation becomes

$$
\rho(\boldsymbol{\xi}, t) H(x, z) J(x, y, z)=\rho(\boldsymbol{\xi}, 0) H(\xi, \zeta)
$$

where $J$ is the Jacobian, $H(x, z)=h_{1}(x, z) h_{3}(x, z)$, and $h_{1}$ and $h_{3}$ are the metric coefficients evaluated on $y=0$ for the coordinates $x$ and $z$ respectively. The condition for a singularity to form is that the Lagrangian gradients of $x$ and $z$ become parallel, i.e.

$$
\nabla_{\boldsymbol{\xi}} x=\lambda, \nabla_{\boldsymbol{\xi}}^{z}
$$

for some constant $\lambda_{1}$.

The physical description of separation given in section 2 is again valid in three dimensions - the separation particle is squashed infinitely thin in the direction of the skewed coordinate $n=x-\lambda, z$. A generalization of the MRS conditions to three-dimensions can also be shown to hold. 


\subsection{Three-Dimensional Asymmetric Separation}

The most general form of separation occurs if the initial singularity develops at a point in the flow where there are no symmetries. An analysis based on expanding the position coordinates $x, z$, and the density $\rho$, in Taylor series can again be performed (VDC). The singularity is found to have a quasi-two-dimensional structure stretched out along the surface line

$$
\bar{x}=\bar{x}_{0}(\bar{z}, t) \equiv \lambda_{\triangleleft} \bar{z}+\lambda_{a}^{(2)} \bar{z}^{2}+\lambda_{\imath}^{(3)} \bar{z}^{3}+\lambda_{a}^{(4)} \delta t \bar{z}
$$

where the origin is taken at the separation particle,

$$
\bar{x}=x-x\left(\boldsymbol{\xi}_{\bullet}, t\right) \quad, \quad \bar{z}=z-z\left(\boldsymbol{\xi}_{\bullet}, t\right)
$$

and the $\lambda_{s}^{(j)}$ are constants that can be calculated from the Taylor series coefficients for $x$ and $z$. The scalings corresponding to $(3.15 c, d)$ are

$$
\bar{x}-\bar{x}_{0}(\bar{z}, t)=|\delta t|^{\frac{3}{2}} X \quad, \quad \bar{z}=|\delta t|^{\frac{1}{2}} Z \quad, \quad y=|\delta t|^{-\frac{1}{4}} Y .
$$

Hence separation occurs in a relatively thin strip of width $|\delta t|^{3 / 2}$ straddling a segment of the oblique, curved separation line $\bar{x}=\bar{x}_{0}(\bar{z}, t)$ of length $\bar{z} \sim|\delta t|^{\frac{1}{2}}$.

Note that once the coordinates have been suitably skewed, the $|\delta t|$ scalings for $X$ and $Y$ are the same as for the two-dimensional case. Moreover, the previous figures $2 d$ and $2 g$ can be interpreted as contours of constant velocity (or density) and constant vorticity respectively for three-dimensional separation after a suitable scaling of the coordinates to remove the dependence on $Z$ (VDC). Similarly, by redefining $\Lambda, m$ and other scaling coefficients, the displacement thickness for three-dimensional separation can be put exactly in to the form (3.19). From such expressions it is possible to calculate contours of constant displacement thickness. Asymptotically they have the form of a crescent shaped ridge. The crescent shape is long and thin because the $\left(\bar{x}-\bar{x}_{0}\right)$ lengthscale is asymptotically shorter than the $\bar{z}$ lengthscale. Figure 3 is an illustration of how contours of displacement thickness might look at a finite time before separation (it was drawn by taking $|\delta t|=0.06$ and unit values for various coefficients - see VDC for further details). We also note that because the separation is quasi-two-dimensional, a 'triple-deck' type interaction develops with essentially the same scalings as before - e.g. see (3.22c). In particular, in the central 
interaction problem, the coordinate $\bar{z}$, which has an interaction length scale $O\left(R e^{-\frac{1}{11}}\right)$, appears only as a parameter.

\subsection{Separation on a Symmetry Line}

While the description in the previous subsection is accurate when separation begins at an asymmetric point in the flow, examples of separation occurring on a symmetry line exist (e.g. Cebeci, Stewartson \& Schimke 1984, Ersoy \& Walker 1987, Xu \& Wang 1988, Wu \& Shen 1990). For instance, separation first develops on a symmetry line in the case of a spheroid at a relatively small, aligned, angle of attack. Yet the separation structure is only a special case of the one described above for this flow, as well as others in which the direction that the separation particle shortens is aligned with the symmetry line.

This changes for another type of symmetric separation, in which the separation particle is being compressed towards the symmetry line. For instance, such finite-time symmetric singularities are generated at the equator of an impulsively rotated sphere (e.g. Banks \& Zaturska 1979), at the apex of an impulsively heated horizontal circular cylinder (Banks \& Campbell 1982, Simpson \& Stewartson 1982b), at the inner bend of a uniformly curved pipe through which fluid starts to flow (Lam 1988), and at the stagnation points on a two-dimensional symmetric body in oscillating flow (Riley \& Vasantha 1989b).

The structure of this type of singularity on the symmetry line was first identified using Eulerian coordinates by Banks \& Zaturska (1979) and Simpson \& Stewartson (1982a), while Van Dommelen $(1981,1990)$ demonstrated that the same results could be recovered by a Lagrangian analysis similar to the one described previously. Further, the simplicity of the Lagrangian approach enabled VDC to extend the description of the singularity structure a little distance off the symmetry line. They were also able to consider a more general form of symmetric separation in which the singularity develops at a point rather than along the entire symmetry line, e.g. as would occur in starting flow through a curved pipe with nonuniform curvature.

This symmetric singularity is not reducible to the asymmetric one, but does have a similar structure. If the coordinates $x$ and $z$ are perpendicular to, and aligned with, the symmetry line respectively, then scalings corresponding to $(3.15 c)$ and $(3.26 a, b)$ involve the same powers of $|\delta t|$. Further, the scaled displacement thickness can again be written in 
terms of a elliptic integral. However, the $y$-position of the separation particle, and hence the displacement thickness, increases more rapidly, in particular as $|\delta t|^{-\frac{1}{2}}$; these scales are illustrated schematically in figure 4.

The most significant difference between this singularity and the asymmetric one concerns the velocity in the central expanding region. For the symmetric singularity this is much larger than the velocity in the upper and lower vorticity layers, whereas the opposite is true for the asymmetric singularity (at least in the frame moving with the singularity). As a result, the pressure gradients induced by the rapidly increasing displacement thickness are first felt in the vorticity layers. Since it is the central layer which is responsible for the growth in boundary-layer thickness, it appears that the first asymptotic rescaling will not lead to an 'interactive' effect that inhibits the development of the singularity. Instead, it is likely that the singularity will continue to be driven by the flow in the central layer, while significant changes occur in the upper and lower layers.

\subsection{Numerical Verification of Symmetry Line Separation}

As indicated above, until a mathematical proof is available, verification of the regularity hypothesis rests on the properties of numerical solutions. One such calculation has been performed by Lam (1988) for impulsively started flow through a uniformly curved circular pipe at large Dean numbers (an idealized model of aortic flow). In suitable nondimensional coordinates, and assuming that the curvature of the pipe is much larger than the radius of the cross section, the governing equations are (e.g. Pedley 1980)

$$
\begin{gathered}
u=\dot{x}, \quad \dot{u}=\sin (x)\left(w_{e}^{2}-w^{2}\right)+D_{y}^{2} u \\
w=\dot{z}, \quad \dot{w}=\dot{w}_{e}+D_{y}^{2} w
\end{gathered}
$$

where $x$ measures distance around the surface of the pipe from the outer bend (the inner bend is at $x=\pi$ ), $z$ measures distance down the pipe, $u$ and $w$ are the corresponding velocities, $w_{e}(t)$ is the inviscid velocity in the central region of the pipe, and $D_{y}$ is the Eulerian derivative. If

$$
\xi=x, \quad \eta=y \quad \text { at } \quad t=0
$$

where $y=0$ defines the surface of the pipe, the Jacobian $J(x, y)$ again satisfies (3.5), while $D_{\nu} u$ is still given by (3.6b). The problem is fully specified by the initial and boundary 
conditions

$$
\begin{gathered}
u=0, \quad w=w_{e} \text { at } t=0+, \\
u=v=w=0 \quad \text { on } \eta=0, \text { and } u \rightarrow 0, w \rightarrow w_{e} \text { as } \eta \rightarrow \infty .
\end{gathered}
$$

These equations have been solved numerically for the choice $w_{0}=1$ by Lam (1988). Following Van Dommelen (1981) coordinate stretches were used in both Lagrangian directions. The one in the $\xi$-direction was chosen so that the tendency of fluid particles to move towards the inner bend, which has the effect of decreasing resolution near the outer bend at larger times, was compensated for by initially skewing the particles towards the outer bend. In the $\eta$ direction a $t^{\frac{1}{2}}$ scaling was used to expand the Rayleigh layer that develops at $t=0+$, and the semi-infinite coordinate range was transformed to $[0,1]$ using an arctan mapping.

The flows on the symmetry lines $\xi=0$ and $\xi=\pi$ were obtained by expanding $x$, $u, w$ in Taylor series in $\xi$ and $(\xi-\pi)$ respectively. The resulting system of parabolic equations for the leading order coefficients depends only on $\eta$ and $t$. These equations were marched forward in time using a second-order finite difference scheme. At each time step the nonlinear difference equations were solved by Newton-Raphson iteration.

Away from the symmetry lines, the governing equations were again discretised by second-order central differences; we note that it was not necessary to skew the finitedifference molecules for the first-order spatial derivatives in $(3.27 b, d)$, as Van Dommelen (1981) needed to do for flow past a circular cylinder (see also Peridier \& Walker 1989). For this two-dimensional problem it was not possible to solve the difference equations by a Newton-Raphson iteration owing to the size of the Jacobian matrix. Instead a modified Alternating Direction Implicit iteration scheme was used in which a partial Newton-Raphson iteration was performed along successive lines of unknowns in the spatial domain. The number of iterations necessary was reduced by first making a 'leapfrog' step to obtain an initial guess for the solution. Lam (1988) gives further details of the method.

Once $x$ was calculated, $y$ was found by numerically solving the Jacobian equation. Due to the singularity that develops, this was done by rewriting (3.5) in the form

$$
\frac{d \eta}{d y}=x_{, \xi}
$$


which is valid on lines of constant $x$. This equation was integrated with a Runge-Kutta scheme using equally spaced steps in $y$; interpolation was used to find $x_{, \xi}$ as necessary.

Figure $5 a$ is a graph of $\min _{\xi, \eta}\left|\nabla_{\xi} x\right|$ against time (in fact, because the separation singularity develops on the symmetry line of the inner bend it is equivalent to a plot of $\min _{\xi=\pi, \eta} x_{, \xi}$. Clearly $\nabla_{\xi} x$ tends to zero linearly in time; we conclude that for impulsively started flow through a curved circular pipe, separation starts at $t=t_{s} \approx 2.813$. The asymptotic scalings predicted for $|\delta t|<<1$ (see figure 4) can be confirmed by plotting a position in the upper vorticity layer as $(y-\eta+c)|\delta t|^{\frac{1}{2}}$ against $(\pi-x)|\delta t|^{-\frac{3}{2}}$, as in figure $5 b$; here $\eta=10$ and $t=2.7,2.75,2.8$ (the constant part $-\eta+c$, with $c=5.5$ was included as a first approximation to account for higher order corrections in the asymptotic expansion for small $|\delta t|)$. The singularity structure is verified because the plots collapse onto each other.

\subsection{Axisymmetric Separation}

Another class of separation singularities is rotationally symmetric about the separation point. For example, singularities develop after a finite time on the axis of a spinning disc or sphere whose direction of rotation is impulsively reversed (Bodonyi \& Stewartson 19.77, Banks \& Zaturska 1981, Stewartson, Simpson \& Bodonyi 1982, Van Dommelen 1987), and at the apex of a sphere which is impulsively heated (Brown \& Simpson 1982, Awang \& Riley 1983).

A Lagrangian analysis similar to that above can be performed for this symmetry as well (VDC). While the precise structure of the separation singularity depends on whether or not the flow has swirl, both types of singularity have qualitative features in common with each other and with the symmetry line singularity described above, e.g. the velocity in the central expanding region is much larger than that in the surrounding vorticity layers. The lateral scaling of the singularity is again $|\delta t|^{\frac{3}{2}}$, but the displacement thickness increases like $|\delta t|^{-1}$ and $|\delta t|^{-\frac{3}{2}}$ for flows with and without swirl respectively (see figure 4 ). The results on the axis can also be obtained by Eulerian analysis (see above references), however the singularity structures slightly off the axis were first obtained by the Lagrangian approach.

\section{Asymmetric Marginal Separation}

\subsection{Introduction}


In the previous section we mentioned the two-dimensional unsteady interaction problem which develops when the pressure perturbations induced by a rapid growth in boundary-layer thickness become too large to be neglected. As an alternative to studying this difficult problem, a formulation in which the interaction is in some sense 'weak' is presented below.

The work in this section was motivated by the observation that if a circular cylinder of diameter $D$ is oscillated perpendicular to its axis through an amplitude $U / \Omega$, then there is a qualitative difference in the flow depending in the size of the Keulegan-Carpenter

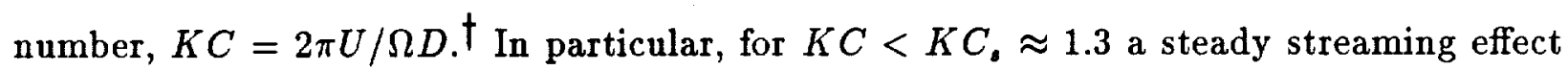
induces boundary-layer collisions at the stagnation points, but no 'vortex shedding' occurs from the surface of the cylinder (e.g. Bearman et al. 1981, Sarpkaya 1986). Note that although such flows have usually been described as separation free, in our terminology the boundary-layer collision is the result of a symmetry line separation; the initial development of this symmetric separation singularity has been studied by Riley \& Vasantha (1989b). For $K C>K C_{\text {, }}$, eddies are shed from the surface of the cylinder, at least while the flow remains two-dimensional (see Tatsuno \& Bearman (1990) and references therein for a more detailed description of this flow and its relevance to offshore structures). For $\left(K C-K C_{0}\right)<<1$, we would expect the vortex shedding to be 'weak', and the question then arises whether a theoretical description of the flow is possible.

In fact from a theoretical standpoint, the problem where the cylinder has been oscillating ad infinitum is not especially attractive because of the complications arising from the boundary-layer collisions at the stagnation points. A natural alternative is to consider the start-up problem, or more generally a case where the cylinder is moved (possibly unidirectionally) only for a finite time. As will be indicated below, the nature of the weak separation for such problems is not as simple as first thought. A more attractive possibility may be the case of separation induced by a vortex impinging on a boundary layer which experiences an otherwise favorable pressure gradient (cf. Doligalski \& Walker 1984). A strong vortex will induce separation, whereas if it is sufficiently weak the flow is expected to be separation free.

For the above flows, the boundary-layer solution depends on a variable parameter

$\dagger$ This is essentially an inverse Strouhal number. 
$a$, (the Keulegan-Carpenter number, the time that the cylinder moves, the strength of the vortex, etc.), in addition to the Lagrangian coordinates and time. We assume that for values of $a$ less than some minimum value, say $a_{a}$, no separation occurs, but that for $a \geq a$. separation does take place. In the spirit of Stewartson, Smith \& Kaups (1982) we call the separation for $a \approx a$, marginal.

Previous studies of unsteady marginal separation include the work of Ruban (1982a), Smith (1982) and Smith \& Elliott (1985). For the most part these authors considered unsteady small perturbations imposed upon flows which were already close to the steady marginal separation condition identified by Ruban (1981, 1982b) and Stewartson et al. (1982). In addition, the timescale of the perturbations was slow compared to the reference time interval $D / U$ (but, see Elliott \& Smith (1987) for a discussion of a shorter timescale problem which may develop subsequently).

Our aim is to provide a theoretical description of marginal separation when the flow starts far from separation, momentarily approaches it, and then recovers to a strongly attached state over an $O(D / U)$ timescale. However, a complication which arises is whether at the critical value of the parameter $a_{\bullet}$, separation occurs at a finite or infinite time. We will show that both cases seem possible, and study possible structures for representative flows.

When the marginal separation occurs at a finite time, we again hypothesize that the classical boundary-layer solution is a regular function of the parameter $a$, as well as being a regular function of the Lagrangian coordinates $(\xi, \eta)$, and the time $t$ :

$$
x=x(\xi, \eta ; t ; a)
$$

On this assumption, we will find a non-interactive description of marginal separation when $a \approx a_{s} ;$ this analysis will then be extended to include interactive effects. As at the start of section 3 the flow is taken to be two-dimensional and incompressible, although generalizations are straightforward.

\subsection{Non-Interactive Asymmetric Marginal Separation}

The analysis of the marginal separation singularity is similar to the two-dimensional asymmetric singularity described in the previous section. As before the existence of a singularity is indicated by the development of a stationary point in $x(\xi, \eta ; t ; a)$ at some 
position $S$ :

$$
x_{\xi}=x_{\eta}=0 \quad \text { at } \quad S=\left(\xi_{1}, \eta_{\bullet}, t_{0}, a_{\mathbf{a}}\right)
$$

In this case the transformations that simplify the Taylor series expansion for $x$ about $S$ are essential, and so these will be described in detail. Similar to the case of non-marginal separation, we shift the origin of the Lagrangian coordinate system to the point $S$, and then rotate the axis system to eliminate the mixed second-order Lagrangian derivative. The shifted and rotated coordinates are denoted by $\left(\hat{k}_{1}, \hat{k}_{2}, \hat{t}, \hat{a}\right)$, and a hat will be used when dependent variables such as $\hat{x}$ are considered a function of these coordinates. As before, if $\hat{t}_{0}$ is the first time that a singularity forms, the first-order derivatives and all but one of the second-order derivatives at $S$ must be zero:

$$
\hat{x}_{\hat{k}_{1}}=\hat{x}_{\hat{k}_{2}}=\hat{x}_{\hat{k}_{1} \hat{k}_{1}}=\hat{x}_{\hat{k}_{1} \hat{k}_{2}}=0 \quad(4.3 a, b, c, d)
$$

The remaining second-order derivative, $\hat{x}_{\hat{k}_{2} \hat{k}_{2}}$, is assumed not to be zero. If it were zero all three second-order derivatives would vanish in the original coordinate system, in addition to the two first order ones. Those five conditions seem too restrictive for four independent coordinates, and they are not required to obtain marginal separation.

Unlike the previous section, a second transformation is now helpful. First, we note that since $\hat{x}_{\hat{k}_{2} \hat{k}_{2}}$ is non-zero, the location where the derivative $\hat{x}_{, \hat{k}_{2}}$ vanishes defines a regular curve $\hat{k}_{2}=\hat{k}_{2,}\left(\hat{k}_{1} ; \hat{t} ; \hat{a}\right)$. We shift the $\hat{k}_{1}$-axis to this curve by the transformation

$$
\hat{\hat{k}}_{2}=\hat{k}_{2}-\hat{k}_{2 s}\left(\hat{k}_{1} ; \hat{t} ; \hat{a}\right)
$$

The result is that the region where the derivative $\hat{\hat{x}}_{, \hat{\hat{k}}_{2}}$ vanishes has been simplified to the $\hat{\hat{k}}_{2}=0$. Hence a singularity occurs when $\hat{\hat{x}}_{\hat{\hat{k}}_{2}}\left(\hat{\hat{k}}_{1}, 0 ; \hat{\hat{t}} ; \hat{\hat{a}}\right)$ vanishes.

The requirement that there is no separation for negative values of $\hat{\hat{a}}$ imposes an additional condition that distinguishes marginal separation from the non-marginal case. In particular, if the derivative $\hat{\hat{x}}_{\hat{k}_{2} \hat{i}}$ were non-zero, a time $\hat{\hat{t}}$ could be found where $\hat{\hat{x}}_{, \hat{\hat{k}}_{1}}(\hat{\hat{k}}, 0 ; \hat{\hat{t}} ; \hat{\hat{a}})$ was zero for small values of $\hat{\hat{a}}$ of both signs, in contradiction of the assumption of marginality. The restriction

$$
\hat{\hat{x}}_{\hat{\hat{k}}_{1} \hat{\hat{t}}}=0
$$


is therefore necessary (and in fact must be so even if $\hat{x}_{\hat{k}_{2} \hat{k}_{2}}$. was zero, since in that case the axis system could be rotated to satisfy (4.5)).

The Taylor series for the streamwise particle position $x$ has thus been simplified to:

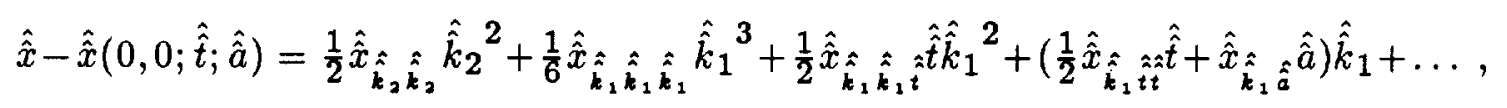

where only those terms which turn out to be significant near the separation particle have been retained. The following requirements on signs are needed in order that there is no separation for negative $\hat{\hat{a}}$ :

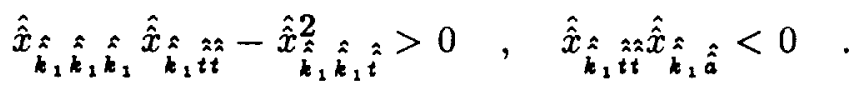

Some care is needed with the first condition, because the second-order time derivatives must satisfy certain restrictions if $\hat{x}$ is to be a solution of the boundary-layer equation; in particular

$$
\hat{x}_{\hat{k}_{1} \hat{t} \hat{t}}=0
$$

To check consistency we note that the transformed derivatives can be expressed in terms of the original ones as:

$$
\begin{array}{r}
\hat{\hat{x}}_{\hat{k}_{1} \hat{k}_{1} \hat{\hat{k}}_{1}}=\hat{x}_{\hat{k}_{1} \hat{k}_{1} \hat{k}_{1}}, \quad \hat{\hat{x}}_{\hat{\hat{k}}_{1} \hat{\hat{k}}_{1} \hat{t}}=\hat{x}_{\hat{k}_{1} \hat{k}_{1} \hat{t}}-\frac{\hat{x}_{\hat{k}_{2} \hat{t}}}{\hat{x}_{\hat{k}_{2} \hat{k}_{2}}} \hat{x}_{\hat{k}_{1} \hat{k}_{1} \hat{k}_{2}} \\
\hat{\hat{x}}_{\hat{k}_{1} \hat{t} \hat{t}}=\hat{x}_{\hat{k}_{1} \hat{t} \hat{t}}-2 \frac{\hat{x}_{\hat{k}_{2} \hat{t}}}{\hat{x}_{\hat{k}_{2} \hat{k}_{2}}} \hat{x}_{\hat{k}_{1} \hat{k}_{2} \hat{t}}+\frac{\hat{x}_{\hat{k}_{2} \hat{t}^{2}}^{2} \hat{x}_{\hat{k}_{2} \hat{k}_{2} \hat{k}_{2}}, \quad \hat{\hat{x}}_{\hat{k}_{1} \hat{\hat{a}}}=\hat{x}_{\hat{k}_{1} \hat{a}}}{\hat{x}_{\hat{k}_{2} \hat{k}_{2}}^{2}}
\end{array}
$$

Since the boundary-layer equation does not restrict the zeroth and first-order time derivatives, it appears that the conditions $(4.6 b, c)$ can, in principle, be met. See the next section for an example where a similar restriction cannot be satisfied.

An important property of the flow for this type of marginal separation can now be deduced from (4.6b) and the expression for the vorticity near the point $S$ at the time of separation $\hat{\hat{t}}=\hat{\hat{a}}=0$ :

$$
\omega \sim-\frac{1}{2} \hat{x}_{\hat{k}_{2} \hat{t}}\left(\hat{\hat{x}}_{\hat{k}_{1} \hat{\hat{k}}_{1} \hat{\hat{k}}_{1}} \hat{k}_{1}^{2}-2 \hat{\hat{x}}_{\hat{k}_{1} \hat{\hat{k}}_{1} \hat{t}} \hat{k}_{1}\left(\frac{\hat{x}_{\hat{k}_{2} \hat{k}_{2}} \hat{k}_{2}}{\hat{x}_{\hat{k}_{2} \hat{t}}}\right)+\hat{\hat{x}}_{\hat{k}_{1} \hat{\hat{t}} \hat{t}}\left(\frac{\hat{x}_{\hat{k}_{2} \hat{k}_{2}}}{\hat{x}_{\hat{k}_{2} \hat{t}}} \hat{k}_{2}\right)^{2}\right)
$$

This expression is everywhere single-signed except at the separation point itself (for definiteness we assume that it is negative), and thus the velocity profile must have a vertical 
inflection point. In addition, because diffusion acts to smooth out the vorticity distribution, we conclude at times before marginal separation, there must be an internal region of positive vorticity, indicated by a negative slope in the velocity profile.

In order to write the results in a standard form we now make a third transformation

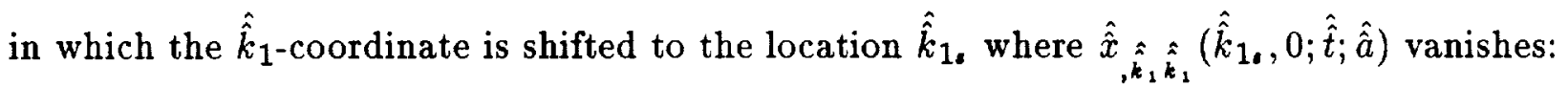

$$
l_{1}=\hat{\hat{k}}_{1}-\hat{\hat{k}}_{1 \cdot}(\hat{\hat{t}} ; \hat{\hat{a}})
$$

We also subtract the motion of the origin from the streamwise position:

$$
\bar{x}=\hat{\hat{x}}-x_{\triangleleft} \quad, \quad x_{s}(\hat{\hat{t}} ; \hat{\hat{a}})=\hat{\hat{x}}\left(\hat{\hat{k}}_{1 \_}, 0 ; \hat{\hat{t}} ; \hat{\hat{a}}\right) \quad
$$

Summarizing the above three transformations, the total transformation consists the initial shift and rotation of the Lagrangian coordinate system followed by:

$$
l_{1}=\hat{k}_{1}-\hat{\hat{k}}_{1 \triangleleft}(\hat{t} ; \hat{a}) \quad, \quad l_{2}=\hat{k}_{2}-\hat{k}_{2 \triangleleft}\left(\hat{k}_{1} ; \hat{t} ; \hat{a}\right) \quad, \quad \bar{x}=x-x,(\hat{t} ; \hat{a}) \quad . \quad(4.11 a, b, c)
$$

These transformations leave the Jacobian unchanged, as well as the Lagrangian expressions for the Eulerian partial derivatives in the boundary layer:

$$
\frac{\partial f}{\partial x}=\left(y_{l_{2}} \frac{\partial f}{\partial l_{1}}-y_{l_{1}} \frac{\partial f}{\partial l_{2}}\right) \quad, \quad \frac{\partial f}{\partial y}=\left(\bar{x}_{, l_{2}} \frac{\partial f}{\partial l_{2}}-\bar{x}_{l_{2}} \frac{\partial f}{\partial l_{1}}\right)
$$

The major effect of the transformation is the change in expression for the convective derivative:

$$
\dot{f}=\frac{\partial f}{\partial \hat{t}}-\hat{\hat{k}}_{1 \triangleleft, \hat{t}} \frac{\partial f}{\partial l_{1}}-\hat{k}_{2,}, \hat{t} \frac{\partial f}{\partial l_{2}}
$$

The advantage of the above transformations is that they significantly simplify the Taylor series expansion for $x$ in the neighborhood of separation:

$$
\bar{x} \sim \frac{1}{2} \bar{x}_{l_{2} l_{2}} l_{2}^{2}+\frac{1}{6} \bar{x}_{l_{1} l_{1} l_{2}} l_{1}^{3}+\left(\bar{x}_{l_{1} \hat{a}} \hat{a}+\frac{1}{2} \bar{x}_{l_{1} \hat{t} \hat{t}} \hat{t}^{2}\right) l_{1}+\ldots
$$

Also, by a suitable choice of the positive $\bar{x}$ and $l_{1}$ directions, and using $(4.6 b, c)$, we can assume

$$
\bar{x}_{l_{2} l_{2}}>0 \quad, \quad \bar{x}_{l_{1} l_{1} l_{1}}>0 \quad, \quad \bar{x}_{l_{1} \hat{t} \hat{t}}>0 \quad, \quad \bar{x}_{l_{1} \hat{a}}<0
$$




\subsection{Non-Interactive Vertical Particle Position}

Next we turn to the non-interactive solution of the continuity equation. This solution is almost identical in form to the one for non-marginal separation derived in the previous section, although the scalings are slightly different. Similar arguments to those leading to (3.15), suggest that an appropriate scaling is

$$
\begin{gathered}
l_{1}=\epsilon L_{1} \quad, \quad l_{2}=\epsilon^{\frac{3}{2}} L_{2} \quad, \quad \hat{t}=\epsilon T, \\
\hat{a}=\epsilon^{2} A \quad, \quad \bar{x}=\epsilon^{3} X \quad, \quad y=\epsilon^{-\frac{1}{2}} Y,
\end{gathered}
$$

where the artificial small parameter $\epsilon$, chosen so that $A=O(1)$, will prove useful when considering the interactive case. We see that the separation region scales as $(\bar{x}, y)=$ $O\left(\hat{t}^{3}, \hat{t}^{-\frac{1}{2}}\right)$, instead of $O\left(\hat{t}^{\frac{3}{2}}, \hat{t}^{-\frac{1}{4}}\right)$ as in $(3.15 c, d)$. Nevertheless, in the limiting process $\epsilon \rightarrow 0$ the scaled displacement effect of the inner region is essentially the same as for the non-marginal case of section 3 :

$$
Y-\frac{1}{2} Y^{+} \sim \pm \int_{L_{1}}^{L_{0}} \frac{d L}{\sqrt{2 \bar{x}_{l_{2} l_{2}}\left\{X-\frac{1}{6} \bar{x}_{l_{1} l_{1} l_{1}} L^{3}-\left(\frac{1}{2} \bar{x}_{l_{1} \hat{t} \hat{t}} T^{2}+\bar{x}_{l_{1} \hat{a}} A\right) L\right\}}}
$$

where, as before, $L_{0}$ is the root of the cubic polynomial in the denominator, the choice is sign is as explained below (3.18), and $\frac{1}{2} Y^{+}(X)$ is the value of the positive integral when $L_{1}=-\infty$. Note that for coefficients satisfying (4.15) solutions can be found for all scaled times $T$ if $A<0$, but that if $A \geq 0$ then a singularity of the form discussed in the previous section develops at $T=-\sqrt{-2 \bar{x}_{i_{1} \hat{a}} A / \bar{x}_{i_{1} \hat{t} \hat{t}}}$

\subsection{Interactive Streamwise Particle Position}

We now turn to the description of the interactive effects induced by the large displacement represented by the scaling $(4.16 f)$. Clearly the asymptotic region that generates the large boundary-layer displacement effect is the rapidly expanding 'central' region in the vicinity of the stationary point. Further, the scalings $(4.16 b, c)$ imply that $\partial / \partial l_{2}>>\left(\partial / \partial \hat{t}, \partial / \partial l_{1}\right)$; thus in that region the convective derivative given by (4.13) can be approximated by the $\partial / \partial l_{2}$ derivative term. In other words, only spatial derivatives remain; due to the relatively rapid motion of the particles through the inner region, the pressure gradient appears quasi-steady to them. 
The first interaction effects are expected to occur when the $O\left(R^{-\frac{1}{2}}|\epsilon|^{-\frac{28}{2}}\right)$ induced pressure gradient (cf. (3.22a)), becomes comparable to the $O(1)$ particle acceleration near the stationary point (cf. (3.22b)). The resulting scalings in the central region are again of the form (4.16) provided the parameter $\epsilon$ is identified as $R^{-\frac{1}{13}}$. However, it is now appropriate to include a pressure expansion of the form

$$
\bar{p} \sim \bar{p}_{0}(\hat{t} ; \hat{a})+\epsilon^{3}\left(\tilde{p}_{\bar{x}} X+P\right),
$$

where $P$ is the pressure disturbance induced by the rapidly growing boundary-layer thickness.

In the central region the leading order asymptotic approximation to the Navier-Stokes $x$-momentum equation is

$$
\hat{k}_{2 \hat{t}_{\hat{i}}}^{2} X_{, L_{2} L_{2}} \sim \hat{k}_{2 \epsilon_{\hat{t}} x_{l_{2} l_{2}}^{2}}^{2}-P_{1} x
$$

where we have identified the constant term from the non-interactive solution which is valid for large negative $T$. The leading order approximation to the normal momentum equation shows that $P$ is again independent of $Y$, while the continuity equation remains

$$
X_{, L_{1}} Y_{, L_{2}}-X_{, L_{2}} Y_{, L_{1}}=1
$$

The first integral to the momentum equation (4.19) is:

$$
X_{, L_{2}}^{2}=2 \bar{x}_{l_{3} l_{2}}\left(X-Q P-C\left(L_{1} ; T ; A\right)\right)
$$

where

$$
Q=2\left(\bar{x}_{l_{2} l_{2}} \hat{k}_{2 s_{\hat{t}}}^{2}\right)^{-1}
$$

Further, it seems that a general functional form for $C$ can be excluded because of the curved topology of the lines of constant $X$ in the Lagrangian domain. In particular, except for special choices of $C$, the limit $L_{2} \rightarrow \pm \infty$ introduces two internal asymptotic regions into the downstream non-interactive boundary layer. One of these regions corresponds to particles emerging from the interaction region, but the other has particles entering it and there is no apparent justification for such an asymptotic structure there. Based on the assumption that the second term in the expansion of the non-interactive region is $O\left(R^{-\frac{1}{2}}\right)$, 
we conclude that the integration constant $C$ which matches the non-interactive solution is given by

$$
C\left(L_{1} ; T ; A\right)=\frac{1}{6} \bar{x}_{l_{1} l_{1} l_{1}} L_{1}{ }^{3}+\left(\frac{1}{2} \bar{x}_{l_{1} \hat{t} \hat{t}} T^{2}+\bar{x}_{l_{1} \hat{a}} A\right) L_{1}
$$

\subsection{Interactive Vertical Particle Position}

With the streamwise particle position known, we can now integrate the continuity equation $(4.20)$ using $(4.21,22,23)$ to find the displacement effect of the central region (cf. $(4.17))$ :

$$
Y-\frac{1}{2} Y^{+} \sim \pm \int_{L_{1}}^{L_{0}} \frac{d L}{\sqrt{2 \bar{x}_{l_{2} l_{2}}\left\{X-Q P-\frac{1}{6} \bar{x}_{l_{1} l_{1} l_{1}} L^{3}-\left(\frac{1}{2} \bar{x}_{l_{1} \hat{t} \hat{t}} T^{2}+\bar{x}_{l_{1} \hat{a}} A\right) L\right\}}}
$$

where $Y^{+}$, etc. again have the same general form as in section 3 . In terms of $(3.19 a)$, the total displacement effect of the central region felt at the top of the boundary layer can be written in the form:

$$
Y^{+}=\frac{1}{\gamma \beta^{\frac{1}{2}}} Y^{+*}\left(\frac{X-Q P}{\alpha \beta^{3}}\right)
$$

where

$$
\alpha=\frac{1}{3} x_{l_{1} l_{1} l_{1}} \quad, \quad \gamma=\sqrt{\frac{1}{3} \bar{x}_{l_{2} l_{2}} \bar{x}_{l_{1} l_{1} l_{1}}}
$$

are constants while

$$
\beta=\sqrt{\frac{\bar{x}_{l_{1} \hat{t} \hat{t}}}{\bar{x}_{l_{1} l_{1} l_{1}}} T^{2}+\frac{2 \bar{x}_{l_{1} \hat{a}}}{\bar{x}_{l_{1} l_{1} l_{1}}} A} .
$$

Since the function $Y^{+*}$ can be stored in table form, the numerical solution of the central region requires no more than table look-up. In contrast, the finite difference solution of the non-marginal central region is awkward because of the irregular behavior of the flow near the edges of the region (Elliott et al. 1983).

\subsection{Bordering Vorticity Layers}

The analysis so far has determined the asymptotic solution in the rapidly expanding region near the stationary point. As in the non-marginal, non-interactive case of section 3 , the boundary-layer particles below the central region form a non-separating vorticity layer at the wall, while those above it form an ejected vorticity layer. 
For the lower vorticity layer, we propose the scalings

$$
l_{1} \quad, \quad l_{2}=l_{2_{l}}\left(l_{1} ; \hat{t} ; \hat{a}\right)+\epsilon^{3} L_{2_{l}} \quad, \quad \bar{x}=\epsilon^{3} X \quad, \quad y \quad, \quad(4.27 a, b, c, d)
$$

in which $l_{2}$ is the marginal but non-interactive prediction for the location where $\bar{x}=0$. These scalings imply that the pressure variations across the wall layer are negligible, while the $O\left(\epsilon^{3}\right)$ velocity perturbations parallel to the wall behave inviscidly. The slip-velocity generated by these perturbations means that a viscous wall layer must also be present with the scalings

$$
l_{1}=l_{1_{v}}(\hat{t} ; \hat{a})+\epsilon^{\frac{3}{2}} L_{1_{v}} \quad, \quad l_{2}=l_{2_{l}}\left(l_{1} ; \hat{t} ; \hat{a}\right)+\epsilon^{3} L_{2_{v}} \quad, \quad \bar{x}=\epsilon^{3} X \quad, \quad y=\epsilon^{\frac{3}{2}} Y_{v} \quad,
$$

where $l_{1}(\hat{t} ; \hat{a})$ is the non-interactive value of $l_{1}$ at the location on the wall where $\bar{x}=0$. No further details of the solution in this region are given since this layer has no leading order effect on the separation processes.

In the upper vorticity layer, an expansion similar to the one for the wall layer holds (cf. $(3.21)$ ),

$$
l_{1} \quad, \quad l_{2}=l_{2_{u}}\left(l_{1} ; \hat{t} ; \hat{a}\right)+\epsilon^{3} L_{2_{u}} \quad, \quad \bar{x}=\epsilon^{3} X \quad, \quad y=\frac{1}{\epsilon^{\frac{1}{3}}} Y^{+}+\tilde{y} . \quad(4.29 a, b, c, d)
$$

The streamwise momentum equation again behaves inviscidly, and the normal momentum equation predicts that the pressure variations across the layer are negligible. In addition the Jacobian shows that variations in $\tilde{y}$ along lines of constant $X$ are $O(1)$, thus confirming that the dominan $\hat{\imath}$ displacement effect at the upper edge of this region is that generated by the $\epsilon^{-\frac{1}{2}} Y^{+}$central region term.

\subsection{Upper Deck}

The large displacement effect at the top edge of the upper vorticity layer leads to a relatively strong, $O\left(R^{-\frac{1}{2}} \epsilon^{-\frac{7}{2}}\right)$, viscous blowing velocity out of the boundary layer. This blowing velocity is significantly reduced in size in another asymptotic region, often called the 'upper deck', above the separating boundary layer. An examination of the scales involved demonstrates that the flow field in this region is irrotational, and hence it is preferable to use Eulerian coordinates. Suitable scalings are

$$
\bar{x}=\epsilon^{3} X \quad, \quad R^{-\frac{1}{2}} y=\epsilon^{3} \tilde{Y},
$$




$$
u=u_{\iota \triangleleft}(\hat{t}, \hat{a})+\epsilon^{3}\left(\tilde{u}_{\tilde{x}} X+\tilde{u}_{\hat{y}} \tilde{Y}+\tilde{U}\right) \quad, \quad R^{-\frac{1}{2}} v=\epsilon^{3}\left(\tilde{v}_{\tilde{y}} \tilde{Y}+\tilde{V}\right),
$$

where $u_{e .}$ is the classical inviscid slip velocity above the separating particle, the linear terms in $X$ and $\tilde{Y}$ are part of the classical irrotational solution (including curvature effects), and the $\tilde{U}$ and $\tilde{V}$ represent the perturbation generated by the displacement effect. From matching to $(4.29 d)$ it follows that

$$
\tilde{V}(X, 0 ; T ; A)=\left(u_{e,}-x_{\iota_{i}}\right) Y_{, X}^{+} \quad \text { on } \quad \tilde{Y}=0,
$$

and we also require that $\tilde{V} \rightarrow 0$ as $\tilde{Y} \rightarrow \infty$. From potential flow theory the blowing velocity (4.31) can be shown to induce a pressure field, which near the wall is given as

$$
P=-\frac{\left(u_{e,}-x_{s_{\hat{t}}}\right)^{2}}{\pi} \int_{-\infty}^{\infty} \frac{Y_{, X}^{+}\left(X^{\prime}\right)}{X-X^{\prime}} d X^{\prime}
$$

\subsection{Result}

The equations $(4.25,26,32)$ and $(3.19)$ form the unsteady marginal interaction problem. By means of the transformation

$$
X=\alpha \beta^{3} \breve{X}, \quad P=\frac{\left(u_{e s}-x_{s_{\hat{t}}}\right)^{2}}{\alpha \beta^{\frac{\gamma}{2}} \gamma} \breve{P}, \quad \mu=\frac{\left(u_{e s}-x_{t \hat{t}}\right)^{2} Q}{\alpha^{2} \beta^{\frac{13}{2}} \gamma},
$$

the nonlinear equation (4.32) can be written in the one parameter form

$$
\breve{P}=-\frac{1}{\pi} \int_{-\infty}^{\infty} \frac{Y_{, \breve{X}^{\prime}}^{+*}\left(\breve{X}^{\prime}-\mu \breve{P}\right)}{\breve{X}-\breve{X}^{\prime}} d \breve{X}^{\prime},
$$

where $\breve{P} \rightarrow 0$ as $|\breve{X}| \rightarrow \infty$. This system is simpler than the interactive problem described by Elliott et al. (1983), and it should be relatively straightforward to find numerical solutions. For the time being we note that while the interaction is expected to change the form of the solution significantly if the scaled parameter $A$ is negative, it still appears, from the definitions of $\beta$ and $\mu$ in $(4.26 c, 33 b)$, that a singularity develops at a finite time if $A$ is positive (cf. (4.25)). This seems to add support to the theory and calculations of Cowley et al. (1988), Smith $(1988,1990)$ and Peridier \& Walker (1989) that outer-deck pressure interactions are not sufficient to eliminate singularities in unsteady separation.

Of course, while the above analysis is consistent to leading order, if it is to be of physical relevance example solutions of the classical boundary-layer equations that demonstrate 
marginal separation must be found, i.e. solutions which satisfy $(4.3,5,6 b, c)$ at the point $S$. Attempts to find such an example for a circular cylinder moved in various monotonic and oscillatory paths have yet to be successful; clearly the mere existence of a certain type behaviour does not necessarily imply that it occurs in all situations. A more promising class of flows might be those induced by vortices; certainly the velocity profiles presented by Peridier \& Walker (1989) for such flows are close to the behaviour required by (4.9).

\section{Large-Time Marginal Symmetric Separation}

\subsection{Introduction}

As outlined in section 3, the possibility of separation at a line of symmetry has been examined by Banks \& Zaturska (1979), Simpson \& Stewartson (1982a) and Riley \& Vasantha $(1989$ b) from an Eulerian standpoint, and by Van Dommelen $(1981,1990)$ and Lam (1988) using a Lagrangian approach.

The question arises whether the symmetry might lead to significant changes in marginal behavior; we will show that it does. In particular we find that symmetric marginal separation is not ordinarily possible at a finite time if the solution to the momentum equations remains regular. We also show that marginal symmetric separation can occur at infinite time, and we propose asymptotic scalings for it.

\subsection{Conditions for Finite Time Marginal Separation}

In the case of symmetric separation, $x$ is an anti-symmetric function of $\xi$ (e.g. Van Dommelen 1990). The development of a singularity is thus characterized by the Lagrangian derivative $x_{, \xi}(\eta ; t ; a)$ vanishing on the symmetry line, i.e.

$$
x_{\xi}=0
$$

since the other first derivative is identically zero by symmetry. The singularity must also occur in the middle of the boundary layer, which implies that the first zero is a minimum in the $x_{, \xi}$ profile, i.e. the first derivative of the profile with respect to $\eta$ must vanish:

$$
x_{\xi \eta}=0
$$

However, if the point $\left(\eta_{\varepsilon} ; t_{s} ; a_{\triangleleft}\right)$ represents the lowest value of $a$ for which a singularity occurs, an additional condition needs to be satisfied. In particular, if $x_{\xi t}$ were nonzero, 
the condition $x_{, \varepsilon}=0$ would define a regular surface $t=t_{\bullet}(\eta ; a)$ with values for both signs of $\left(a-a_{\bullet}\right)$. To avoid this, a necessary condition for regular marginal separation is

$$
x_{\xi t}=0
$$

The Taylor series about the separation point for finite-time marginal separation thus becomes:

$$
x_{, \xi} \sim \frac{1}{2} x_{\xi \eta \eta}\left(\eta-\eta_{\bullet}\right)^{2}+x_{\xi \eta t}\left(\eta-\eta_{\bullet}\right)\left(t-t_{s}\right)+\frac{1}{2} x_{\xi t t}\left(t-t_{\bullet}\right)^{2}+x_{\xi a}\left(a-a_{\triangleleft}\right)+\ldots
$$

Now, in order that there is no separation for $a<a_{\iota}$, the quadratic terms in (5.4) must be definite; yet, if $x$ is to satisfy the boundary-layer equation, the second-order time derivative $x_{\xi t t}$ must be zero (Van Dommelen 1990). That leads to a contradiction, and we conclude that (5.4) cannot describe marginal separation. Hence, either the initial separation time must approach infinity when the parameter $a$ reaches its critical value, or the structure must become singular in Lagrangian coordinates. A possible exception occurs if $x_{\xi \eta t}$ also vanishes, so that the marginality depends on higher order derivatives. However, there seems no justification to expect this derivative to be zero at the same point that $(5.1,2,3)$ are satisfied.

\subsection{Large Time Marginal Structure}

To establish what happens when the separation becomes marginal at a symmetry line, numerical computations were conducted for impulsively started front stagnation point flows. After some trial and error it was decided to concentrate on a rigid body which is started with unit velocity, decelerated, accelerated again and then kept at unit velocity. More precisely, we prescribed the streamwise gradient $G=\partial u_{c} / \partial x$ of the external flow velocity at the front stagnation point as

$$
\begin{aligned}
& G=(1-a)+a \cos (\Omega t), \quad \text { for } 0 \leq \Omega t \leq 2 \pi \quad ; \\
& G=1 \quad, \quad \text { for } 2 \pi \leq \Omega t .
\end{aligned}
$$

Numerical solutions were obtained using Lagrangian coordinates since these give a precise definition of when separation starts. Preliminary runs suggested that the computational times would be minimised by setting $\Omega=1.1$ (see also Riley \& Vasantha, 1989b). In 
figure $6 a$ we have plotted the separation times as a function of $a$ for this choice of $\Omega$. Separation was found to occur for $a>a_{\star} \approx 0.879$. In the same graph we have also plotted the time, say $t_{m}$, at which $x_{, \xi}$ attained its (positive) minimum for $a<a_{.}$. The apparent symmetry of the figure about $a_{d}$ is true to graphical accuracy, which allows a relatively precise estimate of $a$, to be made. Note that both times rapidly increase as $a_{*}$ is approached. Further evidence that these times become infinite for $a=a$, is given in figure $6 \mathrm{~b}$ which is a plot of $t_{m}$ and $t_{1}$ against $\ln \left|a-a_{\bullet}\right|$. There seems to be a linear dependence (in fact $t_{\mathbb{a}} \approx-\frac{1}{2} \ln \left|a-a_{\bullet}\right|+$ constant), in agreement with an infinite time of marginal separation.

A naive attempt to obtain an asymptotic description of the marginal singularity using exact solutions to the inviscid momentum equation, as was done by Van Dommelen (1981) for the non-marginal case, appears to fail here. However, careful examination of the numerical solutions suggested a possible asymptotic structure for $a=a_{a}$. This turns out to have significant regions of steady flow. Since it is usually simpler to describe these in Eulerian coordinates, for the rest of this section we revert to the Eulerian form for the equations of motion:

$$
\begin{gathered}
g_{t}+g^{2}+v g_{y}=G_{t}+G^{2}+g_{v y}, \\
g+v_{y}=0
\end{gathered}
$$

where $g$ is the streamwise gradient of the velocity on the symmetry plane.

First we recall that the standard symmetric separation singularity has a negative local minimum for $g$ that approaches negative infinity as $t \rightarrow t$, (e.g. Banks \& Zaturska 1979). However, because the second derivative of a function is non-negative near a minimum, some a priori estimates restrict the possibility of separation. In particular, if the value of the minimum for $g$ is at or above $-G$ once $G$ is positive, the minimum value must increase with respect to the external flow value $G$, so that separation cannot occur. The marginal case seems to occur when the minimum of $g$ approaches $-G$ from below - this value, (here -1 ), is indeed a stationary position of the inviscid part of the momentum equation (5.6a).

For the asymptotic region within which $g$ is close to -1 , there are a range of asymptotic scalings which select only the steady, inviscid terms from the momentum equation at leading order. The precise scaling is fixed by the condition that the unsteady and viscous 
correction terms do not introduce singularities in the expansion at the minimum in the velocity profile. This requires that both correction terms appear at the same order in the asymptotic expansion. After some algebra these considerations lead to the following scalings and solution for the expanding central region:

$$
\begin{gathered}
t-t_{0}=\tau, \quad y-\beta_{0}=\tau^{\frac{3}{2}} Y \\
g \sim \cos (Y)-\tau^{-1} \frac{1}{2}+\tau^{-\frac{3}{2}} \beta_{1} \sin (Y)-\tau^{-2} \frac{3}{8}(\cos (Y)+Y \sin (Y)) \\
v \sim-\tau^{\frac{1}{2}} \sin (Y)+\tau^{-\frac{1}{2}} \frac{1}{2} Y+\tau^{-1} \beta_{1} \cos (Y)+\tau^{-\frac{3}{2}} \frac{3}{8}(2 \sin (Y)-Y \cos (Y))
\end{gathered}
$$

where $\beta_{0}$ and $\beta_{1}$ are constants defined below, and $t_{0}$ is a constant which is chosen to eliminate a complementary function in the first-order terms. Note that the undetermined constants which appear in each term of $(5.7 c, d)$ when the relevant governing equations are solved, are fixed either by matching with the Hiemenz layer adjacent to the wall (see below), or by the requirement that the term one order $\tau^{-1}$ smaller is free of singularities at $Y=\pi$, i.e. where $g \approx-1$.

Near the wall, a perturbed Hiemenz front stagnation point boundary-layer profile exists:

$$
v \sim v_{0}(\hat{y})+\tau^{-1} v_{1}(\hat{y})+\tau^{-2} v_{2}(\hat{y})+\tau^{-3} v_{3}(\hat{y})+\cdots
$$

where

$$
\hat{y}=y-\beta_{0},
$$

and $\beta_{0} \approx 0.64790$ is the Hiemenz displacement thickness. Runge-Kutta solutions of the governing equations for the $v_{j}$, using the following asymptotic behaviour for large $y$,

$$
\begin{gathered}
v_{0} \sim-\hat{y}, \\
v_{1} \sim \frac{1}{6} \hat{y}^{3}+\frac{1}{2} \hat{y}+\beta_{1}, \\
v_{2} \sim-\frac{1}{120} \hat{y}^{5}-\frac{1}{2} \beta_{1} \hat{y}^{2}+\frac{3}{8} \hat{y}+\beta_{2}, \\
v_{3} \sim \frac{1}{5040} \hat{y}^{7}+\frac{1}{24} \beta_{1} \hat{y}^{4}+\frac{1}{16} \hat{y}^{3}-\left(\frac{3}{4} \beta_{1}+\frac{1}{2} \beta_{2}\right) \hat{y}^{2}+\left(\frac{3}{4}+\frac{1}{2} \beta_{1}^{2}\right) \hat{y}+\beta_{3},
\end{gathered}
$$

yield

$$
\beta_{1}=0.10285 \quad, \quad \beta_{2}=0.0821, \quad \beta_{3}=0.200,
$$


and the normalized wall shear gradient as

$$
g_{v} \sim 1.232588-\tau^{-1} 0.473524-\tau^{-2} 0.32339-\tau^{-3} 0.58698
$$

As in the preceding sections, above the central expanding region there is an upper vorticity layer present, although here the velocity is only slightly perturbed from the $G=1$ external flow (unlike the finite velocity variations of sections $3 \& 4$ ). We again use the transposition theorem

$$
\tilde{y}=y-y_{\text {ref }}(t) \quad, \quad \tilde{v}=v-\dot{y}_{\text {ref }}(t),
$$

where

$$
y_{\mathrm{ref}}(t) \sim 2 \pi \tau^{\frac{1}{2}}+\beta_{0}+\tau^{-1} \beta_{1}-\tau^{-\frac{3}{2}} \frac{3}{4} \pi+\cdots,
$$

to subtract off the appropriate upward motion of the viscous layer. With this choice of $y_{\text {ref }}$ the upper viscous layer expands in integer inverse powers of $\tau$ :

$$
\begin{gathered}
\tilde{v} \sim-\tilde{y}+\tau^{-1} \tilde{v}_{1}+\cdots \\
\tilde{v}_{1} \sim \alpha_{1}\left\{\left(\frac{1}{6} \tilde{y}^{3}+\frac{1}{2} \tilde{y}\right) \int_{\tilde{y}}^{\infty} \frac{e^{-\frac{1}{2} \gamma^{2}}}{\left(\gamma^{2}+1\right)^{2}} d \gamma-\int_{\tilde{y}}^{\infty}\left(\frac{1}{6} \gamma^{3}+\frac{1}{2} \gamma\right) \frac{e^{-\frac{1}{2} \gamma^{2}}}{\left(\gamma^{2}+1\right)^{2}} d \gamma\right\},
\end{gathered}
$$

where

$$
\alpha_{1} \sim\left\{\int_{-\infty}^{\infty} \frac{e^{-\frac{1}{2} \gamma^{2}}}{\left(\gamma^{2}+1\right)^{2}} d \gamma\right\}^{-1}
$$

Finally, in order to complete the asymptotic solution. it is necessary to consider one more region, because far above the upper vorticity layer the exponentially small disturbances in the almost potential flow are determined by the initial solution (cf. Brown \& Stewartson 1965, Van Dommelen \& Shen 1985). We therefore introduce an adjustment region where

$$
\tilde{y}=e^{\tau} \tilde{Y} \quad, \quad \tilde{v}=-\tilde{y}-e^{-w} \quad, \quad w \sim e^{2 \tau} \frac{1}{2} \tilde{Y}^{2}+4 \tau+W_{2}(\tilde{Y})+\ldots \quad . \quad(5.14 a, b, c)
$$

The function $W_{2}$ is not fixed by substituting into the momentum equation, and may depend on the time evolution. However, for small $\mathrm{Y}$, matching with $(5.12,13)$ yields

$$
W_{2} \sim 4 \ln (\tilde{Y})+\ln (-\ln \tilde{Y})-\ln \left(\frac{1}{2} \alpha_{1}\right)
$$


while for large $\tilde{Y}$ an analysis similar to that of Van Dommelen \& Shen (1985) gives

$$
W_{2} \sim 2 \ln (Y)+b_{00}
$$

where $b_{00}$ is a constant.

The large time marginal structure derived here seems self-consistent and agrees qualitatively with numerical data. However, the Lagrangian numerical computation is very ill-conditioned near marginality, which makes a quantitative comparison impossible.

\section{Concluding Remarks.}

Much of what we know today about the computed and analytical structure of the initial stages of unsteady separation has resulted from the introduction of Lagrangian coordinates to the problem. While these coordinates have their well known disadvantages (e.g. non-uniqueness of the coordinate system, lengthy formulae, lack of a steady state), in the case of unsteady separation these are outweighed by some of their less publicized advantages (e.g. simplified convection, particle accumulation in the separation region, adaptation to inviscid boundary-layer thickening, decoupling of the streamwise and normal particle positions).

In numerical work, a Lagrangian solution for unsteady boundary-layer separation problems is advantageous because the resolution problems for more conventional procedures are severe. Lagrangian procedures work well for such flows: the first accepted solution to unsteady two-dimensional separation was Lagrangian, (Van Dommelen \& Shen 1980), and since then many other flows have been calculated by this method. Yet based on our own experiences and those of others, it appears that while most ordinary numerical techniques will work for Lagrangian coordinates, (e.g. Crank-Nicolson, SOR, LSOR, ADI, multigrid iteration, approximate factorization, multi-level time discretisations, etc.), they have to be applied with care. Despite such difficulties Lagrangian coordinates are possibly the best generally applicable numerical method for obtaining solutions to the boundarylayer equations when separation occurs. It might also be argued that the common tendency to concentrate on Eulerian coordinates has been at the expense of the development of more robust procedures to overcome the difficulties that arise in Lagrangian computations, e.g. varying coefficients and the need to regenerate the mesh adaptively. A current emphasis is 
on three-dimensional unsteady computations, in order to verify the existence of the threedimensional separation structure (see subsection 3.8). For that reason, we have developed a three-dimensional Lagrangian code; this uses overlapping meshes, local discretizations, and ADI iteration to resolve some of the topological issues inherent in three-dimensional curved surfaces.

For analytical work, the advantages of a Lagrangian approach are due to one key observation; namely that in Lagrangian coordinates the components of particle position parallel to the surface are governed by momentum equations which decouple from the continuity equation that specifies the particle position normal to the surface. In part, this decoupling occurs because the pressure gradient which drives the flow is independent of the normal coordinate. As a result of the Lagrangian simplifications, a general analytic theory of unsteady separation can be obtained by hypothesizing that it is only the normal component of particle position which becomes singular, i.e. by assuming that the other components of particle position remain regular (a conjecture supported, for example, by numerical solutions). On this basis, the self-consistency of the asymptotic expansions to arbitrary high order is clear. More important is the indeterminacy in the asymptotic expansions which arises because of a certain arbitrariness as to which Lagrangian derivatives vanish at separation. In the present approach the indeterminacy is removed by assuming that the smallest possible number of Lagrangian derivatives are zero, while satisfying any conditions imposed by symmetries. This assumption is clearly plausible, and is supported by numerical solutions.

In principle, it should also be possible to explain the same separation structures using Eulerian coordinates; indeed some of the symmetry line singularities were first obtained this way. However, the complexity of the Eulerian analysis rapidly increases with the number of dimensions. As a result, even the expansion for two-dimensional asymmetric separation have not been taken to sufficiently high-order to determine uniquely an arbitrary function that arises in the analysis. In addition, no purely Eulerian description has been given either of the general three-dimensional separation structure or of the form of separation off a symmetry line or axis.

We note that because of the simplicity of the Lagrangian approach, this method of solution has been applied to a class of problems closely related to the symmetry-line singu- 
larity; namely exact solutions to the two-dimensional Navier-Stokes equations, or the threedimensional Euler equations, with symmetry-line, stagnation-point similitude (e.g. Stern \& Paldor (1983), Russell \& Landahl (1984), Stuart (1988, 1989), Childress et al. (1989)). The important point to note about these equations is that the pressure-gradient term in the $x$-momentum equation which governs the flow towards the symmetry line, is dependent only on the time, $t$; in particular it is independent of the normal co-ordinate, $y$. Hence, for a flow that is unbounded as $y \rightarrow \infty$, in Lagrangian coordinates the $x$-momentum equation decouples from the continuity equation and the two other momentum equations (cf. classical boundary-layer theory); in 'channel flow' the $x$-momentum equation and the continuity equation are coupled through the boundary condition to fix the unknown pressure gradient.

We recall that for inviscid flows Van Dommelen (1981) has proved that $x$ is a regular function of Lagrangian coordinates, provided that the streamwise pressure gradient is regular. Hence for flows unbounded in $y$, for which it is normal to prescribe a regular pressure-gradient, the ordinary inviscid singularity has the Banks \& Zaturska (1979) structure. For examples of more general flows leading to the same singularity see Stern \& Paldor (1983), Russell \& Landahl (1984), Stuart (1988, 1989), and Childress et al. (1989). Further, as the numerical boundary-layer solutions of Van Dommelen (1987), Lam (1988) and Dennis \& Ingham (1979) show, these boundary-layer collisions also occur when the domain is bounded in the $x$-direction (in this case the stagnation-point, similitude equations arise from a Taylor series expansion about the symmetry line).

Childress et al (1989) present, in addition, inviscid 'channel-flow' solutions for which the pressure gradient is given implicitly and can become infinite within a finite time; this allows alternative singular behaviour. However, attempts to find equivalent finite-time singularities in the viscous equations have not yet proved successful (Cox 1989, Budd, Dold, \& Stuart 1990), especially for a geometry bounded in $y$.

As an illustration of the simplicity of the Lagrangian approach, the interactive structure of a new form of asymmetric, marginal separation has been derived. The flow is assumed to approach, and then recover from, separation on the same timescale as it takes for a fluid particle to pass over the body; interactive effects are only important for a short $O\left(R^{-\frac{1}{13}}\right)$ timescale. We find that a 'triple-deck' type interaction can modify, but appar- 
ently not remove, the separation singularity. Although verification of the existence of this type of marginal separation awaits the numerical solution of the boundary-layer equations, we envision that the solution will be applicable for flows which start far from separation, momentarily approach it, and then recover to a strongly attached state, e.g. the temporary approach of a vortex to a boundary layer, or a pitching airfoil.

We have also shown that finite-time marginal separation cannot occur on a symmetry line, although it is possible at infinite time. This suggests the possibility of a second type of asymmetric marginal separation which can occur at infinite time.

Finally, we note that it has been argued (e.g. Ersoy \& Walker 1985, Stuart 1988) that the explosive growth associated with boundary-layer separation singularities may be related to eruptions from the sub-layer of a turbulent boundary layer, and so with the regeneration of vorticity in such layers. In particular Ersoy \& Walker $(1985,1987)$ and Hon \& Walker (1988) have studied the boundary-layer flow induced by two of the proposed basic elements of wall-layer turbulence, namely the hairpin vortex (e.g. Head \& Bandyophadyay 1981) and the discrete loop vortex (e.g. Falco 1977). Inter alia, they show that a hairpin vortex with a symmetry plane can induce a separation singularity behind the vortex head. As yet the boundary-layer calculations are confined to the symmetry line, and there is no guarantee that the hairpin vortex initiates separation there; indeed twodimensional approximations for the flow generated by the counter-rotating hairpin-vortex legs suggest that initial separation singularities off the symmetry line are possible (e.g. Ersoy \& Walker 1985). However, if the initial separation singularity is not a symmetric boundary-layer collision then the region of boundary-layer growth will be crescent-shaped (VDC); as J.D.A. Walker (1989, private communication) has noted, this is in qualitative agreement with experimental observations.

A complementary view of the eruptions from the sub-layer is that the spanwise motion induced by the vortices can lead to symmetry-line, boundary-layer collisions (e.g. Stuart 1988). However, further theory and calculations are needed to determine whether either or both types of separation are indeed responsible for sub-layer bursting and related phenomena (e.g. the formation of secondary hairpin vortices).

It has also been suggested that separation singularities may be related to boundarylayer transition, for instance through the spanwise flow induced by longitudinal vortices 
(e.g. Stuart 1965). Here we refer to the important work of Hall \& Smith (1989), and references therein, concerning vortex/wave interactions. These authors derive nonlinear modulation equations of novel type, and propose, inter alia, singular terminal forms of the solutions at finite times or finite distances downstream. Such singularities lead to the rapid shortening of time and lengthscales - a phenomena typical of transition. We finish by noting that for some of the modulation equations, especially those of boundary-layer type, a Lagrangian approach may be advantageous in determining which of the proposed singularities, and thus which physical processes, are of acceptable form.

This work was supported in part by the Applied Mathematical Sciences section of the Department of Energy (DE-AS03-76ER72012 KC-07-01-01), the US Army Research Office through the Mathematical Sciences Institute of Cornell University, the SERC, the Air Force Office of Scientific Research (AFOSR F49620-89-C-0014), and the National Aeronautics and Space Administration (NAS1-18605) through the Institute for Computer Applications in Science and Engineering, NASA Langley Research Centre. SJC is grateful to Robert McLachlan and Philip Saffman for helpful discussions concerning the numerical results in section 5 .

\section{References}

Awang, M.A. Omar \& Riley, N. 1983 Unsteady free convection from a heated sphere at high Grashof number. J. Engng. Math. 17, 355-365.

Banks, W.H.H. \& Zaturska, M.B. 1979 The collision of unsteady laminar boundary layers. J. Engng. Math. 13, 193-212.

Banks, W.H.H. \& Zaturska, M.B. 1981 The unsteady boundary-layer development on a rotating disc in counter rotating flow. Acta Mechanica 38, 143-155.

Banks, W.H.H. \& Campbell, M. 1982 The unsteady collision of free-convective boundary layers. SIAM J. Appl. Math. 42, 1217-1230.

Bearman, P.W., Graham, J.M.R., Naylor, P. \& Obasaju, E.D. 1981 The role of vortices in oscillatory flow about bluff bodies. Int. Symp. Hydrodynamics in Ocean Engineering, Norw. Inst. Tech. 621.

Bodonyi, R.J. \& Stewartson, K. 1977 The unsteady laminar boundary layer on a rotating disk in a counter-rotating fluid. J. Fluid Mech. 79, 669-688. 
Bouard, R. \& Coutenceau, M. 1980 The early stage of development of the wake behind an impulsively started cylinder for $40<R e<10000$. J. Fluid Mech. 101, 583-607.

Brown, S.N. \& Stewartson, K. 1965 On similarity solutions of the boundary-layer equations with algebraic decay. J. Fluid Mech. 23, 673-687.

Brown, S.N. \& Simpson, C.J. 1982 Collision phenomena in free-convective flow over a sphere. J. Fluid Mech. 124, 123-127.

Budd, C.J., Dold, J.W. \& Stuart, A.M. 1990 A study of blow-up in systems of PDEs with conserved first integral. In Preparation.

Cebeci, T. 1979 The laminar boundary layer on a circular cylinder started impulsively from rest. J. Comp. Phys. 31, 153-172.

Cebeci, T. 1986 Unsteady boundary layers with an intelligent numerical scheme. J. Fluid Mech. 163, 129-140.

Cebeci, T., Stewartson, K. \& Schimke, S.M. 1984 Unsteady boundary layers close to the stagnation region of slender bodies. J. Fluid Mech. 147, 315-332.

Childress, S., Ierley, G.R., Spiegel, E.A. \& Young, W.R. 1989 Blow up of unsteady twodimensional Euler and Navier-Stokes solutions having stagnation-point form. J. Fluid Mech. 203, 1-22.

Collins, W.M. \& Dennis, S.C.R. 1973 Flow past an impulsively started circular cylinder. J. Fluid Mech. 60, 105-127.

Cowley, S.J. 1983 Computer extension and analytic continuation of Blasius' expansion for impulsive flow past a circular cylinder. J. Fluid Mech. 135, 389-405.

Cowley, S.J. \& Smith, F.T. 1985 On the stability of Poiseuille-Couette flow: a bifurcation from infinity. J. Fluid Mech. 156, 83-100.

Cowley, S.J., Duck, P.W. \& Tutty, O.R. 1988 On the numerical integration of the unsteady triple-deck boundary-layer equations. Presentation at soth BTMC, Imperial College London, April 1988.

Cowley, S.J. \& Van Dommelen, L.L 1990 Genesis of three-dimensional separation. Workshop on Analytical Methods in Unsteady Separation, Ohio State University, January 1990. Cox, S. 1990 A similarity solution of the Navier-Stokes equations for two-dimensional flow 
in a porous-walled channel. Ph.D. Thesis, University of Bristol.

Dennis, S.C.R. \& Ingham, D.B. 1979 Laminar boundary layer on an impulsively started rotating sphere. Phys. Fluids 22, 1-9.

Didden, N. \& Ho, C.-M. 1985 Unsteady separation in a boundary layer produced by an impinging jet. J. Fluid Mech. 160, 235-256.

Doligalski, T.L. \& Walker, J.D.A. 1984 Boundary layer induced by a convected twodimensional vortex. J. Fluid Mech. 138, 1-28.

Elliott, J.W., Cowley, S.J. \& Smith, F.T. 1983 Breakdown of boundary layers: (i) on moving surfaces; (ii) in semi-similar unsteady flow; (iii) in fully unsteady flow. Geophys. Astrophys. Fluid Dynamics 25, 77-138.

Elliott, J.W. \& Smith, F.T. 1987 Dynamic stall due to unsteady marginal separation. $J$. Fluid Mech. 179, 489-512.

Ersoy, S. \& Walker, J.D.A. 1985 Viscous flow induced by counter-rotating vortices. Phys. Fluids 28, 2687-2698.

Ersoy, S. \& Walker, J.D.A. 1987 The boundary-layer due to a three-dimensional vortex loop. J. Fluid Mech. 185, 569-598.

Falco, R.E. 1977 Coherent regions in the outer region of a turbulent boundary layer. Phys. Fluids 20, S124-S132.

Ghia, K.N. (ed.) 1987 Forum on unsteady flow separation. ASME, New York.

Hall, P. \& Smith, F.T. 1989 On strongly nonlinear vortex/wave interactions in boundarylayer transition. ICASE Rep. 89-82.

Head, M.R. \& Bandyophadyay, P. 1981 New aspects of turbulent boundary-layer structure. J. Fluid Mech. 107, 297-337.

Henkes, R.A.W.M. \& Veldman, A.E.P. 1987 On the breakdown of the steady and unsteady interacting boundary-layer description. J. Fluid Mech. 179, 513-530.

Hon, T.-L. \& Walker, J.D.A. 1988 Evolution of Hairpin Vortices in a Shear Flow. NASA Tec. Mem. 100858

Ingham, D.B. 1984 Unsteady separation. J. Comp. Phys. 53, 90-99.

Lam, S.T. 1988 On high-Reynolds-number laminar flows through a curved pipe, and past 
a rotating cylinder. Ph.D. dissertation, Univ. of London.

Matsushita, M., Murata, S. \& Akamatsu, T. 1984 Studies on boundary-layer separation in unsteady flow using an integral method. J. Fluid Mech. 149477.

Moore, F.K. 1958 On the separation of the unsteady laminar boundary layer. In BoundaryLayer Research, ed. H.G. Gortler. Springer.

Nagata, H., Minami, K. \& Murata, Y. 1979 Initial flow past an impulsively started circular cylinder. Bull. JSME 22, 512-520.

Nagata, H., Funada, H., Kawai, K. \& Matsui, T. 1985 Unsteady flows in the region behind a circular cylinder started impulsively. Bull. JSME 28, 2599.

Pedley, T.J. 1980 The fluid mechanics of large blood vessels. Cambridge University Press. Pepin, F. 1990 Simulation of the flow past an impulsively started cylinder using a discrete vortex method. Ph.D. Thesis, California Institute of Technology.

Peridier, V.J. \& Walker, J.D.A. 1989 Vortex-induced boundary-layer separation. Lehigh University Rep. FM-13

Prandtl, L. 1904 Uber Flüssigkeitsbewegung bei sehr kleiner Reibung. In Ludwig Prandtl gesammelte Abhandlüngen, Springer-Verlag 1961, 575-584.

Puppo, G. 1990 Prandtl's equations: numerical results about singularity formation and a new numerical method. Ph.D. Thesis, New York University.

Riley, N. \& Vasantha, R. 1989a Unsteady high-Reynolds-number flows. J. Fluid Mech. 205, 337.

Riley, N. \& Vasantha, R. 1989b An unsteady stagnation point flow. Q. J. Mech. Appl. Math. 42, 511-521.

Rosenhead, L. (ed.) 1963 Laminar boundary layers. Oxford University Press (Also Dover, 1988).

Rott, N. 1956 Unsteady viscous flows in the vicinity of a separation point. Q. Appl. Math. 13, 444-451.

Ruban, A.I. 1981 Singular solution of boundary-layer equations which can be extended continuously through the point of zero surface friction. Izv. Akad. Nauk. SSSR, Mekh. Zhid. Gaza No. 6, 42-52. Trans. in Fluid Dyn. 16, 835-843. 
Ruban, A.I. 1982a Stability of the preseparation boundary layer on the leading edge of a thin airfoil. Izv. Akad. Nauk. SSSR, Mekh. Zhid. Gaza No. 6, 55-63. Trans. in Fluid Dyn. $17,860-867$.

Ruban, A.I. 1982b Asymptotic theory of short separation regions on the leading edge of a slender airfoil. Izv. Akad. Nauk. SSSR, Mekh. Zhid. Gaza No. 1, 42-51. Trans. in Fluid Dyn. 17, 33-41.

Russell, J.M. \& Landahl, M.T. 1984 The evolution of a flat eddy near a wall in an inviscid shear flow. Phys. Fluids 27, 557-570.

Sarpkaya, T. 1986 Force on a circular cylinder in viscous oscillatory flow at low KeuleganCarpenter numbers. J. Fluid Mech. 165, 61.

Sears, W.R. 1956 Some recent developments in airfoil theory. J. Aeronaut. Sci. 23, 490-499.

Sears, W.R. \& Telionis, D.P. 1975 Boundary-layer separation in unsteady flow. SIAM J. Appl. Math. 23, 215.

Shen, S.F. 1978a Unsteady separation according to the boundary-layer equation. Adv. Appl. Mech. 13, 177.

Shen, S.F. 1978 b Unsteady separation of three-dimensional boundary layers from the Lagrangian viewpoint. In Nonsteady Fluid Dynamics, eds. D. E. Crow \& J.A. Miller, 47-51. ASME, New York.

Shen, S.F. \& Wu, T. 1988 Unsteady separation over maneuvering bodies. AIAA paper 88-3552-CP.

Simpson, C.J. \& Stewartson, K. 1982a A note on a boundary-layer collision on a rotating sphere. Z. Angew. Math. Phys. 33, 370-378.

Simpson, C.J. \& Stewartson, K. 1982b A singularity in an unsteady free-convection boundary layer. Q. J. Mech. Appl. Math. 35, 291-304.

Simpson, R.L. 1989 Turbulent boundary-layer separation. Ann. Rev. Fluid Mech. 21, 205-234.

Smith, F.T. 1982 Concerning dynamic stall. Aeronaut. Q. 33 331-352.

Smith, F.T. 1986 Steady and unsteady boundary-layer separation. Ann. Rev. Fluid Mech. 
$18,197-220$.

Smith, F.T. 1988 Finite-time breakup can occur in any unsteady interacting boundary layer. Mathematika 35, 256-273.

Smith, F.T. 1990 Finite-time singularities in unsteady separation. Workshop on Analytical Methods in Unsteady Separation, Ohio State University, January 1990.

Smith, F.T. \& Elliott, J.W. 1985 On the abrupt turbulent reattachment downstream of leading-edge laminar separation. Proc. R. Soc. Lond. A401, 1-27.

Smith, F.T., Papageorgiou, D. \& Elliott, J.W. 1984 An alternative approach to linear and nonlinear stability calculations at finite Reynolds numbers. J. Fluid Mech. 146, 313-330

Stern, M.E. \& Paldor N. 1983 Large amplitude long waves in a shear flow. Phys. Fluids 26, 906-919.

Stewartson, K., Simpson, C.J. \& Bodonyi, R.J. 1982 The unsteady boundary layer on a rotating disk in a counter rotating fluid II. J.Fluid Mech. 121, 507-515.

Stewartson, K., Smith, F.T. \& Kaups, K. 1982 Marginal separation. Stud. Appl. Math. 67, 45-61.

Stuart, J.T. 1965 The production of intense shear layers by vortex stretching and convection. $A G A R D$ Rep. 514.

Stuart, J.T. 1988 Nonlinear Euler partial differential equations: singularities in their solution. In Proc. Symp. Honor of C.C. Lin, eds. D.J. Benney, Chi Yuan, F.H. Shu, 81-95. World Sci. Pub., Singapore.

Stuart, J.T. 1989 The Lagrangian picture of fluid motion and its implication for flow structures.

Ta Phuoc Loc \& Bouard, R. 1985 Numerical solution of the early stage of the unsteady viscous flow around a circular cylinder: a comparison with experimental visualisation and measurements. J. Fluid Mech. 160, 93.

Tatsuno, M. \& Bearman, P.W. 1990 A visual study of the flow around an oscillating circular cylinder at low Keulegan-Carpenter numbers and low Stokes numbers. J. Fluid Mech. 211, 157-182.

Telionis, D.P. \& Tsahalis, D.Th. 1974 Unsteady laminar separation over impulsively moved 
cylinders. Acta Astronautica 1, 1487.

Tutty, O.R. \& Cowley, S.J. 1986 On the stability and the numerical solution of the unsteady interactive boundary-layer equation. J. Fluid Mech. 168, 431-456.

Van Dommelen, L.L. 1981 Unsteady boundary-layer separation. Ph.D. dissertation, Cornell University.

Van Dommelen, L.L. 1987 Computation of unsteady separation using Lagrangian procedures. In Proc. of the IUTAM Symp. on Boundary-Layer Separation, eds. F.T. Smith \& S.N. Brown, 73-87. Springer-Verlag.

Van Dommelen, L.L. 1990 On the Lagrangian description of unsteady boundary layer separation. Part 2. The spinning sphere. J. Fluid Mech. 210, 627-645.

Van Dommelen, L.L. \& Cowley, S.J. 1990 On the Lagrangian description of unsteady boundary layer separation. Part 1. General theory. J. Fluid Mech. 210, 593-626.

Van Dommelen, L.L. \& Shen, S.F. 1980 The spontaneous generation of the singularity in a separating laminar boundary layer. J. Comp. Phys. 38, 125-140.

Van Dommelen, L.L. \& Shen, S.F. 1982 The genesis of separation. In Numerical and Physical Aspects of Aerodynamic Flows, ed. T. Cebeci. Springer-Verlag.

Van Dommelen, L.L. \& Shen, S.F. 1983a Boundary-layer separation singularities for an upstream moving wall. Acta Mech. 49, 241-254.

Van Dommelen, L.L. \& Shen, S.F. 1983b An unsteady interactive separation process. AIA A J. 21, 358-362.

Van Dommelen, L. L. \& Shen, S. F. 1985 The flow at a rear stagnation point is eventually determined by exponentially small values of the velocity. J. Fluid Mech. 157, 1-16.

Van Dyke, M. 1975 Perturbation methods in fluid mechanics. The Parabolic Press, Stanford, CA, 86.

Wang, K.C. 1979 Unsteady boundary-layer separation. Martin Marietta Lab., Baltimore, Maryland, USA, Tech. Rept. MML TR 79-16c.

Williams, J.C. 1977 Incompressible boundary-layer separation. Ann. Rev. Fluid Mech. 9, 113.

Wu, T. 1989 Ph.D. dissertation, Cornell University. 
Wu, T. \& Shen, S.F. 1990 A multi-zone time-marching technique for unsteady separating three-dimensional boundary layers, and its application to the symmetry-plane solution of an impulsively-started prolate spheroid. ASME Int. Sym. on Nonsteady Fluid Dyn. Toronto, June 1990.

Xu, W.C. \& Wang, K.C. 1988 Unsteady laminar boundary layer along the symmetry plane of an impulsively started prolate spheroid. J. Fluid Mech. 195, 413-435. 


\section{Figure 1a}
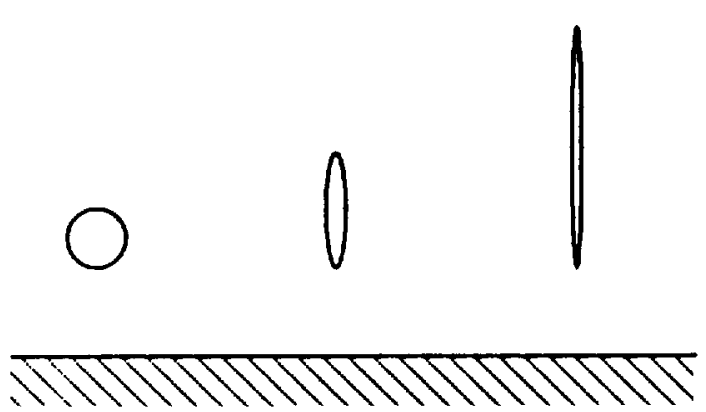

Figure 1b

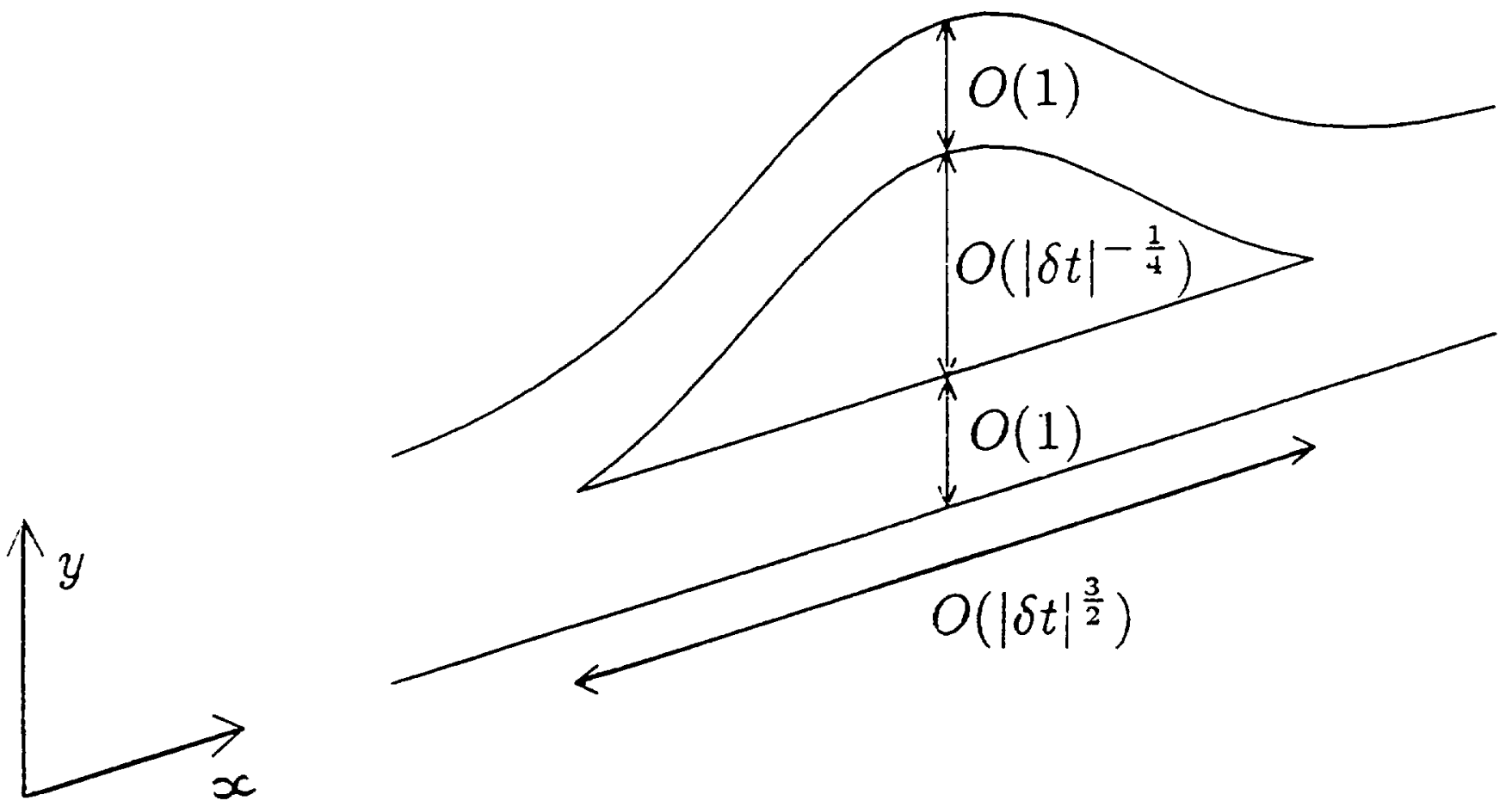

Figure 1. Mechanics of unsteady separation (schematic). (a) The particle deformation which gives rise to separation. (b) Separation of the boundary layer into sublayers due to the particle deformation. 


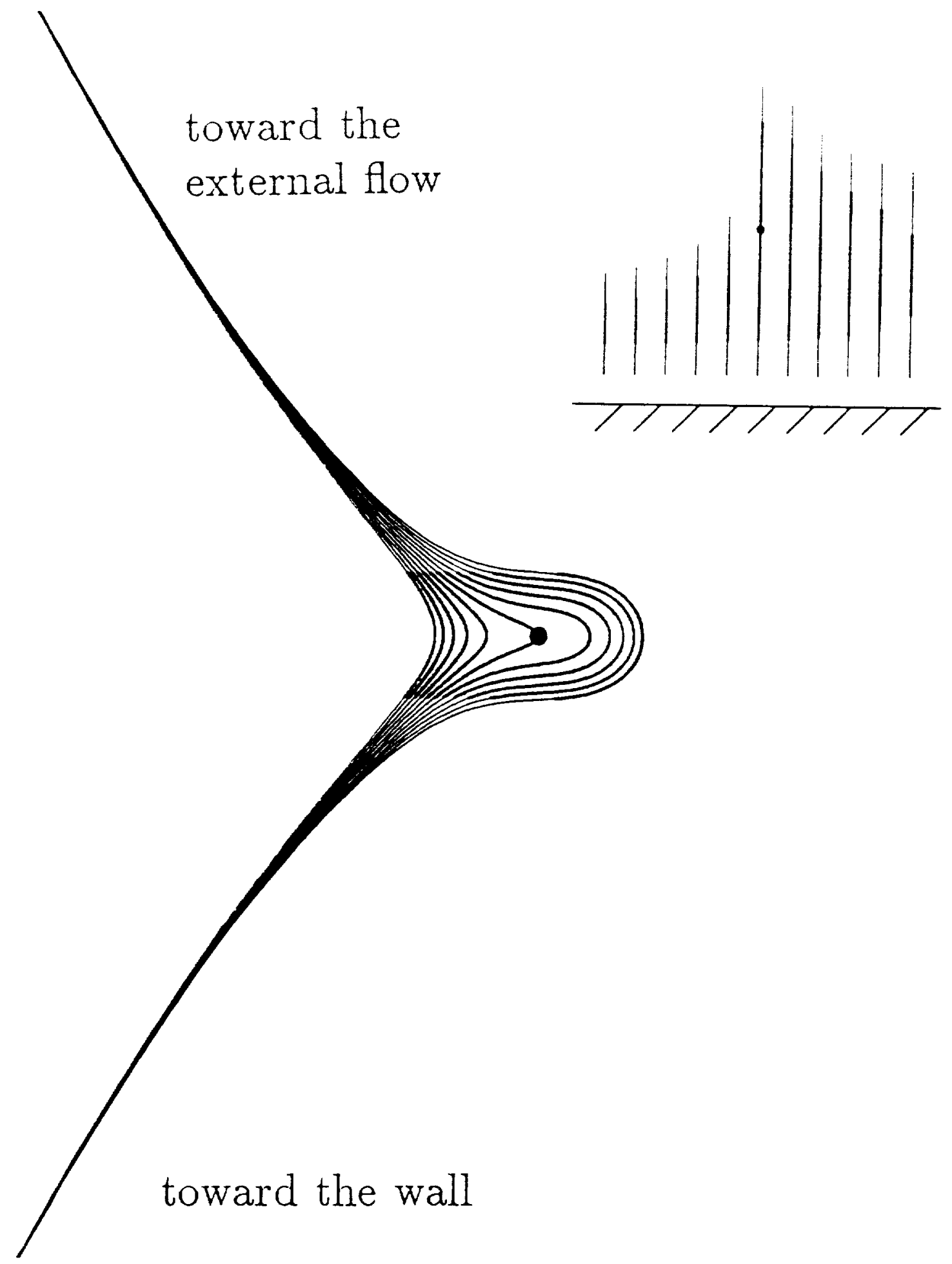

Figure 2. Structure of asymmetric two-dimensional unsteady separation. (a) Lines of constant particle position $x$ in the Lagrangian domain, and in the physical domain (inset). 


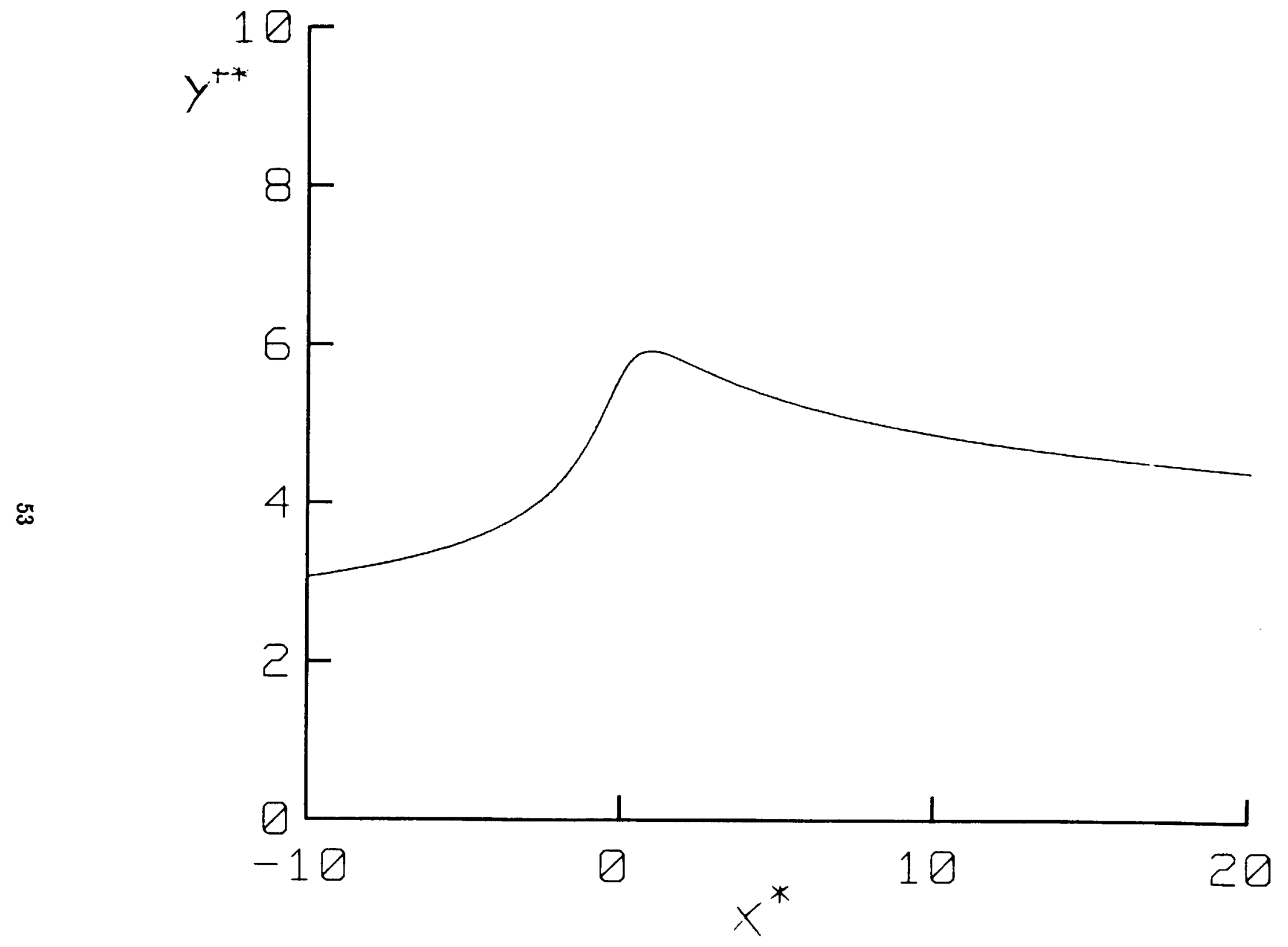

Figure 2. Structure of asymmetric two-dimensional unsteady separation. (b) Scaled and normalized boundary-layer thickness near unsteady separation. 


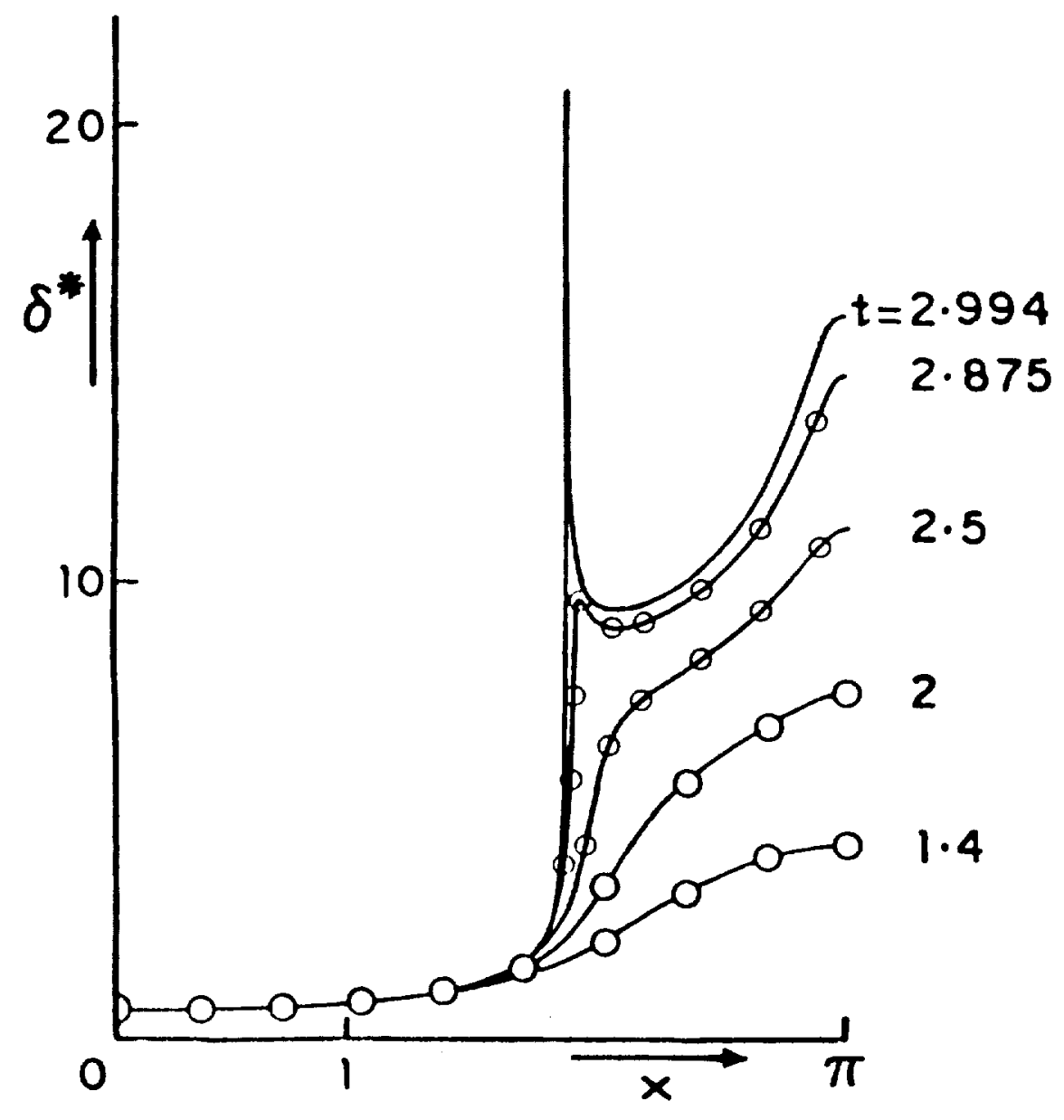

Figure 2. Structure of asymmetric two-dimensional unsteady separation. (c) Computed boundary-layer displacement thickness, $\delta^{*}$, for an impulsively started circular cylinder. o: coarse grid; $\bigcirc$ : Cebeci (1979). 


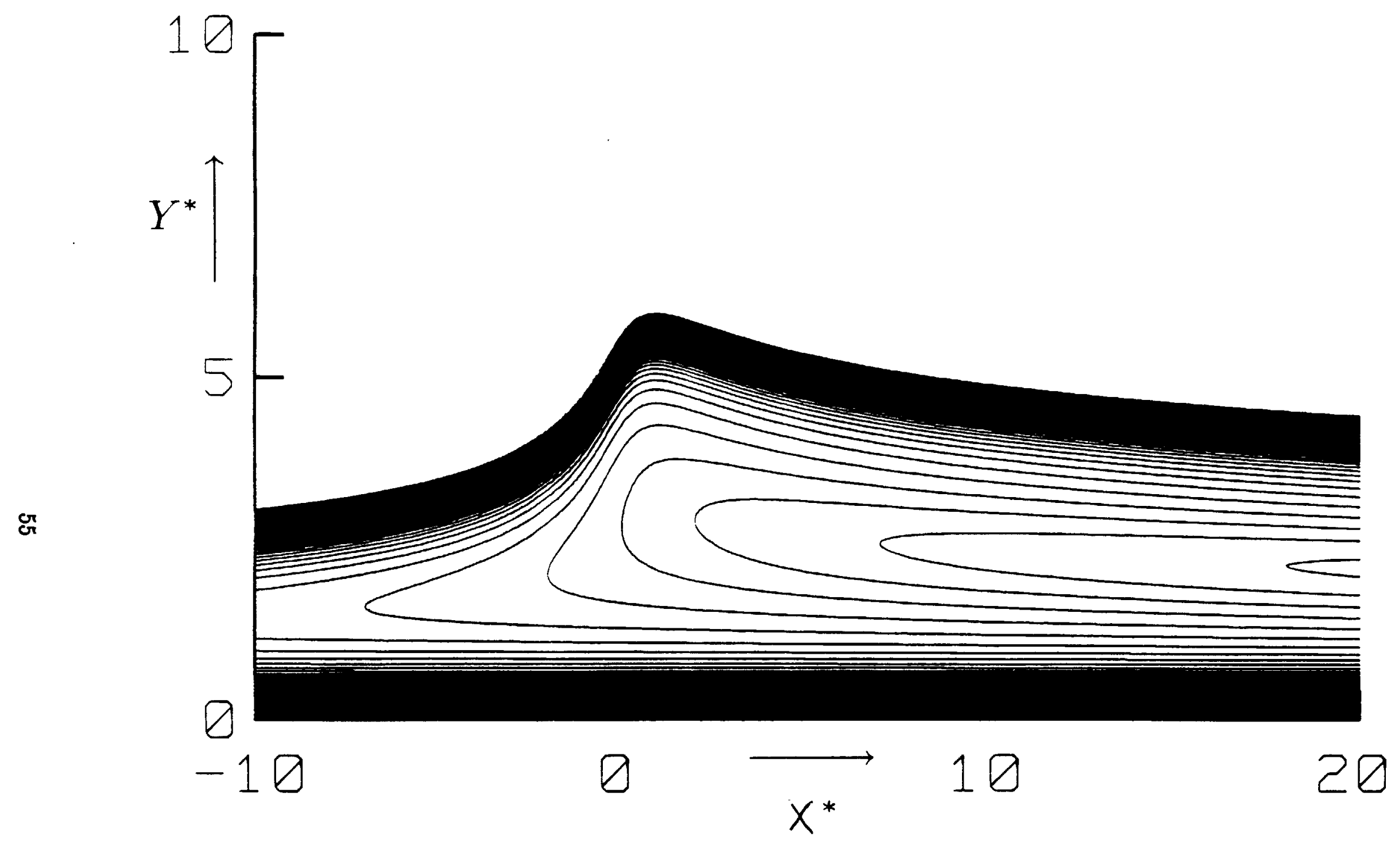

Figure 2. Structure of asymmetric two-dimensional unsteady separation. (d) Scaled and normalized shape of the lines of constant velocity near unsteady separation. 


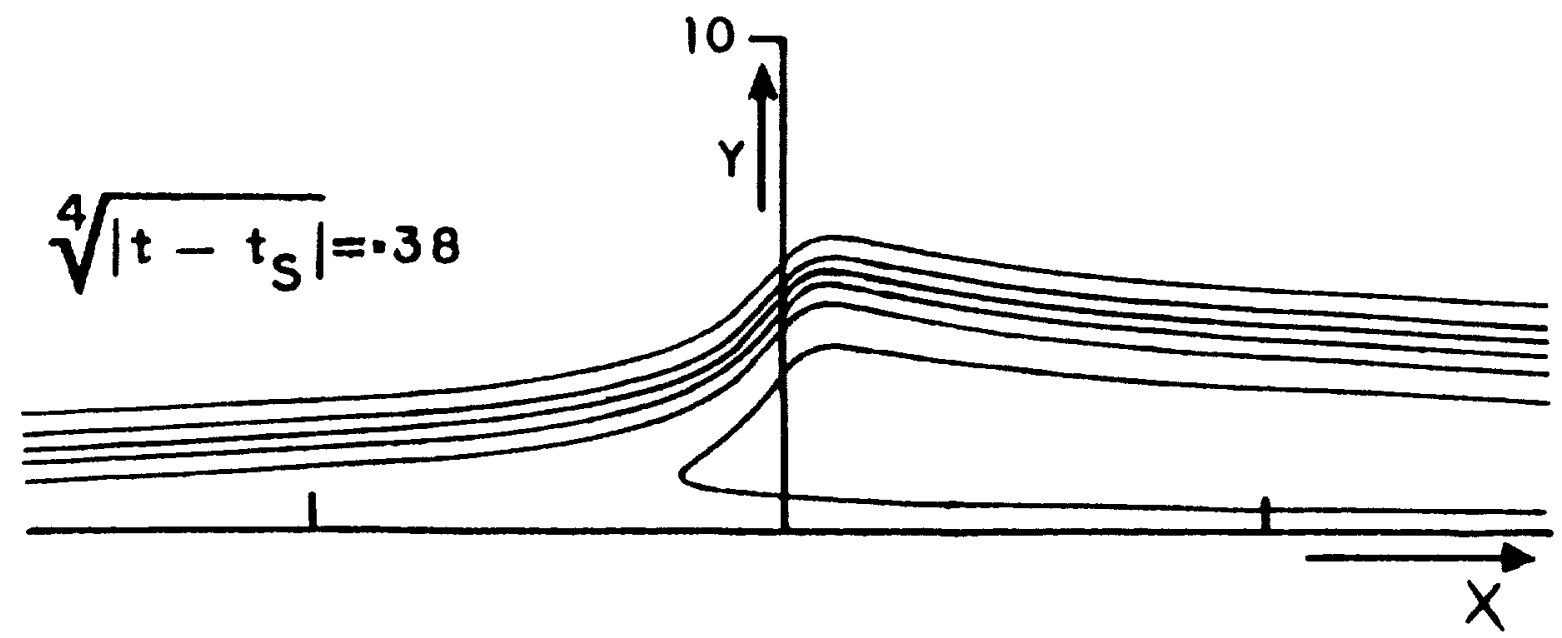

Figure 2. Structure of asymmetric two-dimensional unsteady separation. (e) Computed lines of constant velocity for an impulsively started circular cylinder.
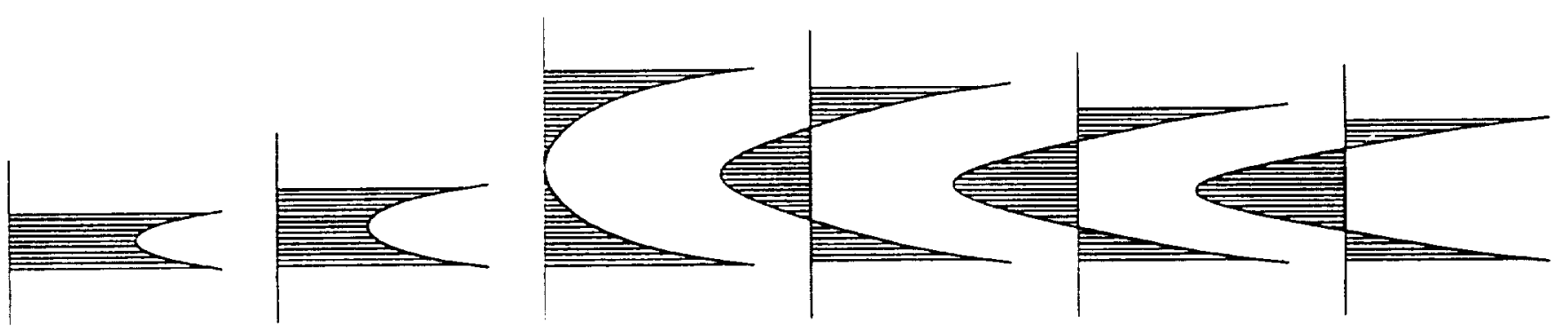

Figure 2. Structure of asymmetric two-dimensional unsteady separation. $(f)$ Shape of the velocity profiles near the separation particle in the middle of the boundary layer. 


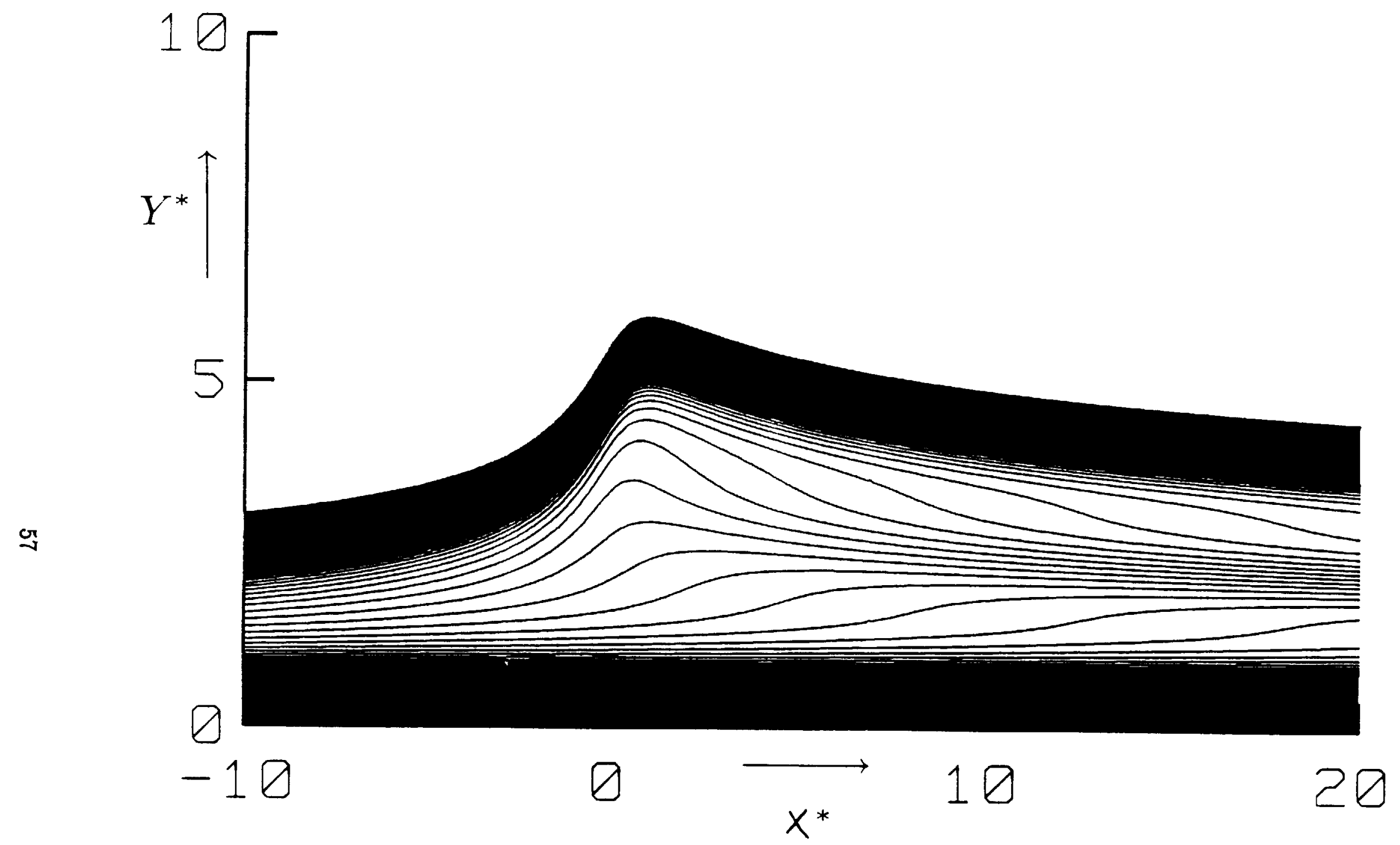

Figure 2. Structure of asymmetric two-dimensional unsteady separation. ( $g$ ) Scaled and normalized shape of the lines of constant vorticity near unsteady separation. 


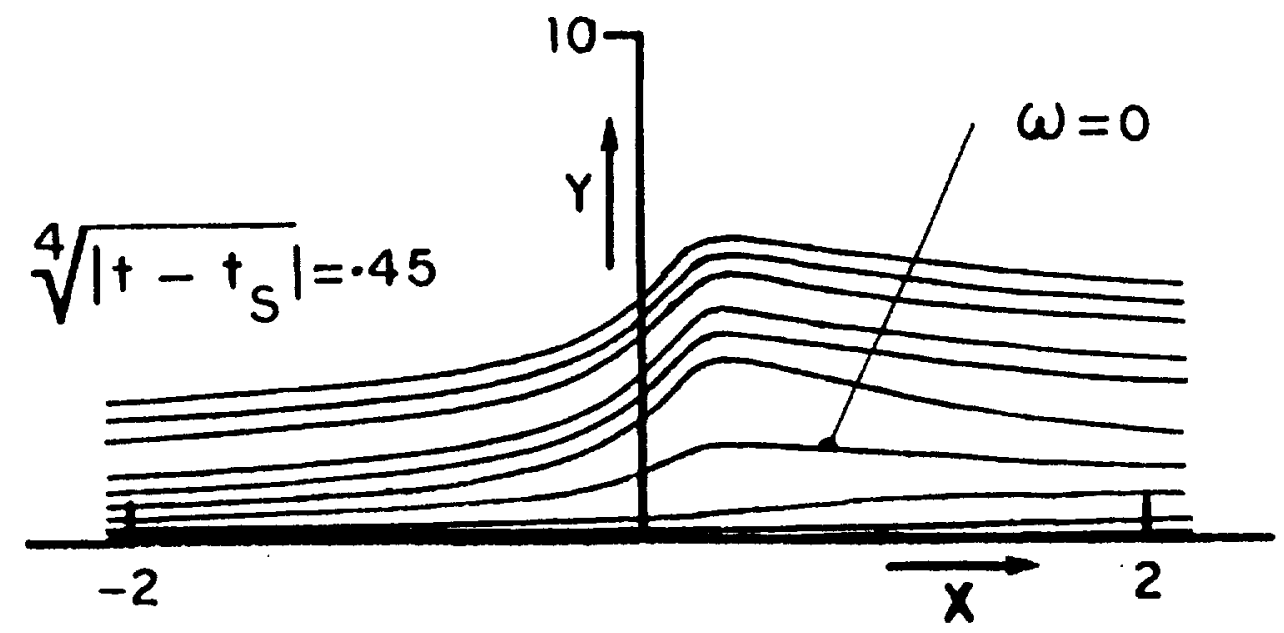

Figure 2. Structure of asymmetric two-dimensional unsteady separation. ( $h$ ) Computed lines of constant vorticity for an impulsively started circular cylinder. 

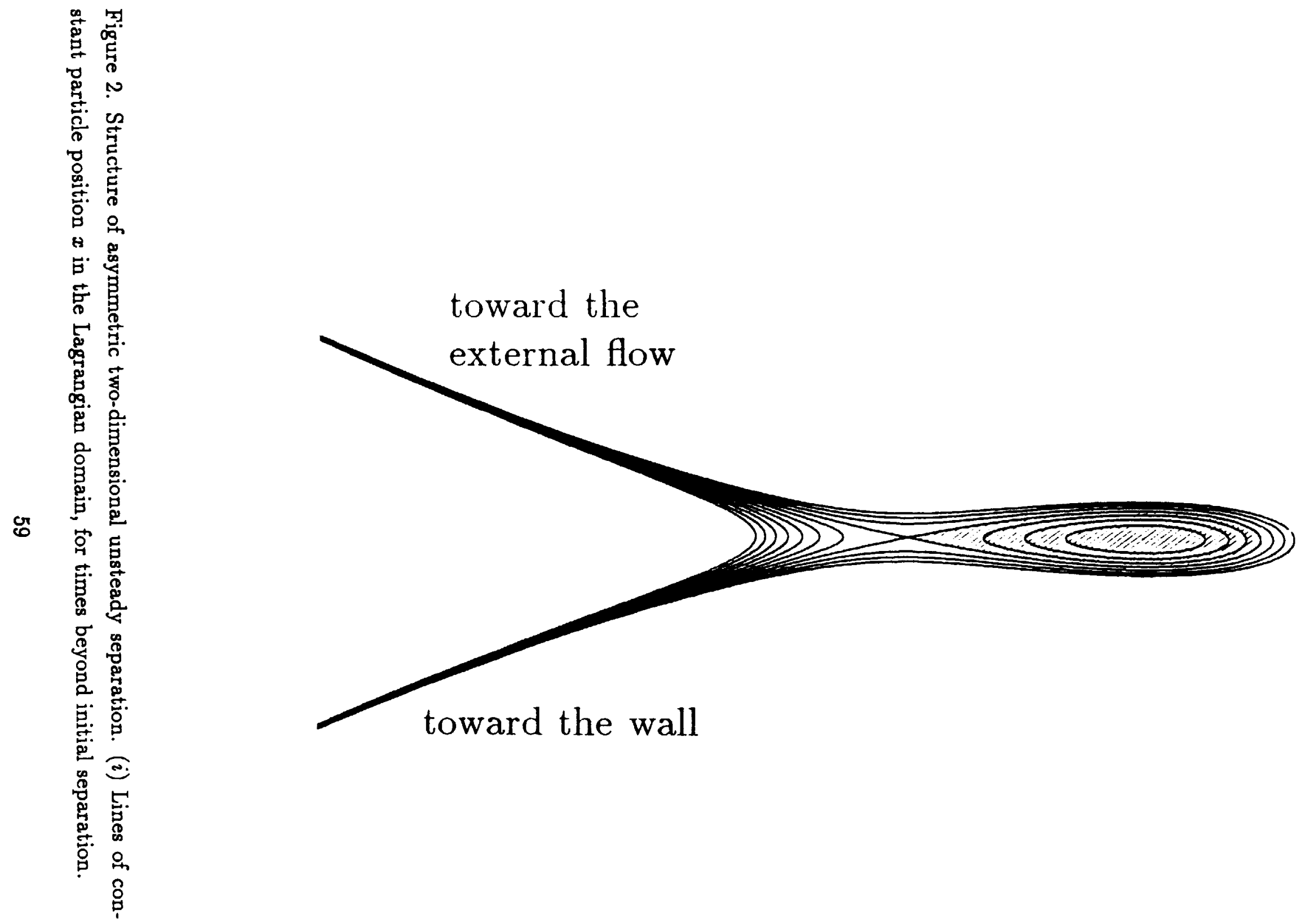


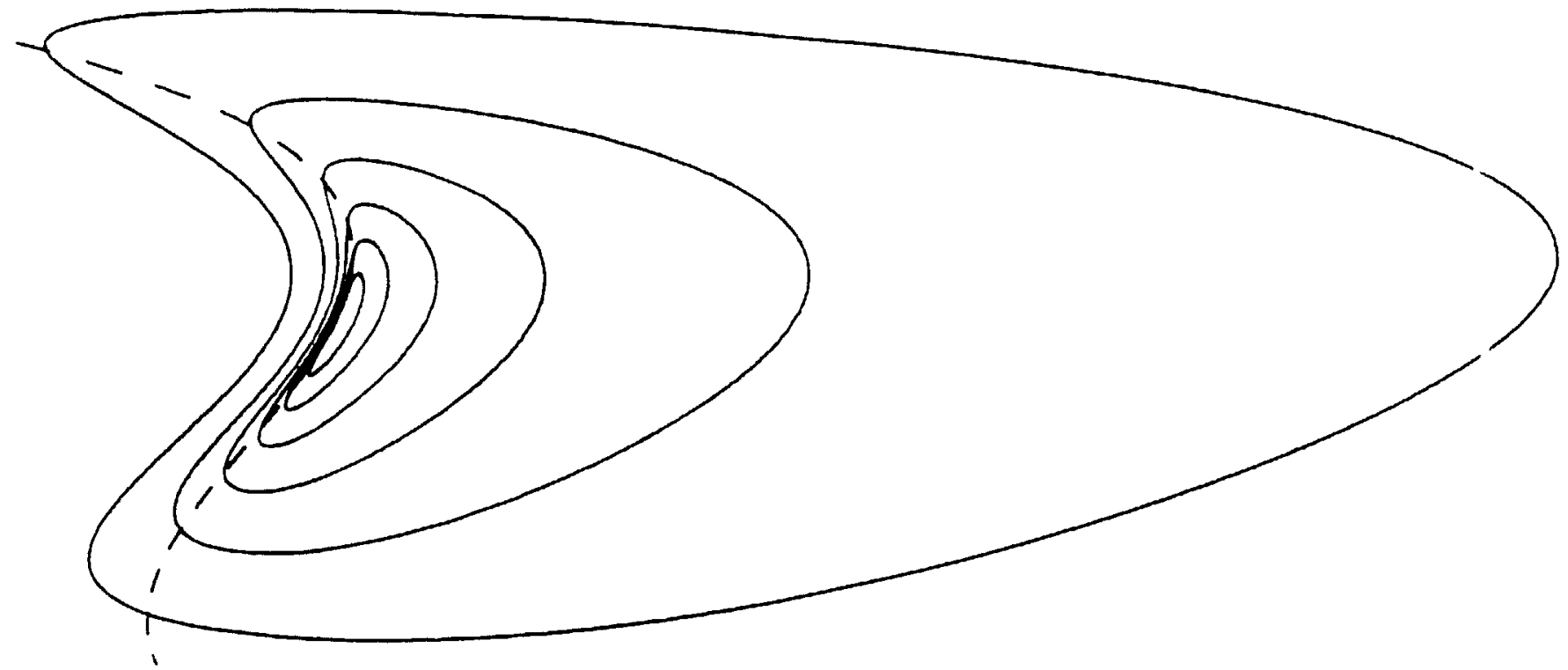

Figure 3. Schematic contours of constant boundary-layer thickness in three-dimensional asymmetric flow. 


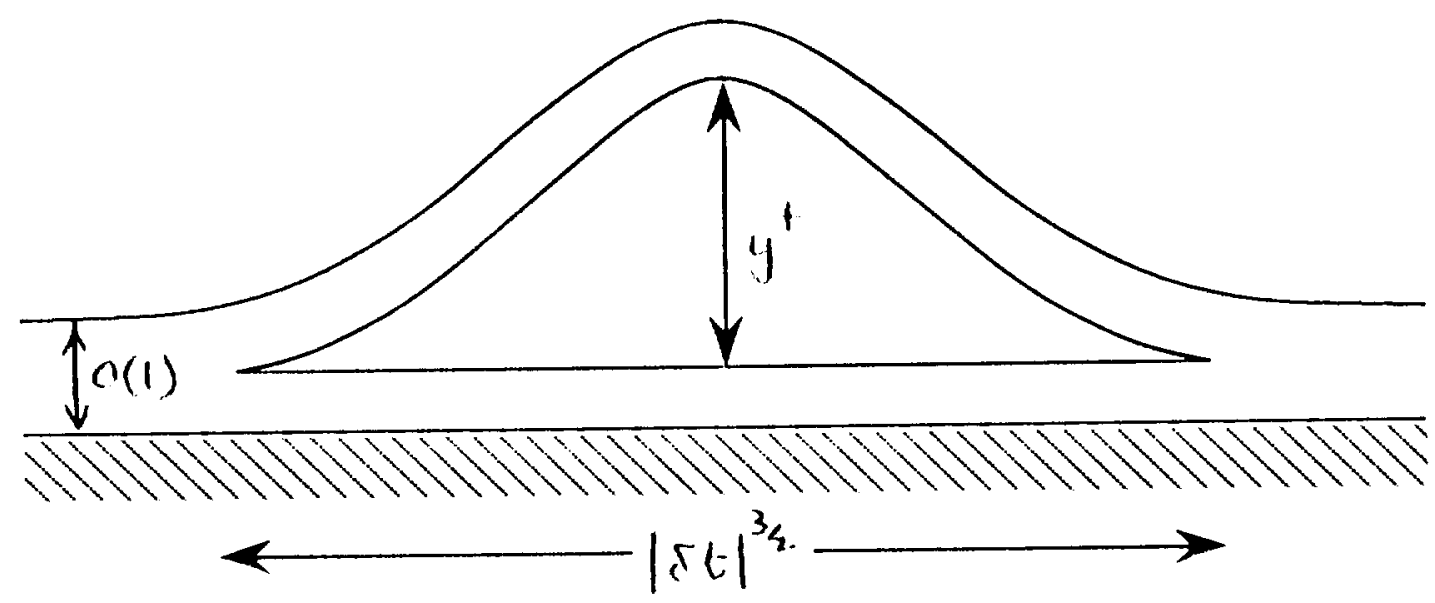

Figure 4. Schematic of asymptotic regions for unsteady boundary-layer collision. At a symmetry line $y^{+} \sim|\delta t|^{-\frac{1}{2}}$; at an axis for flow with swirl $y^{+} \sim|\delta t|^{-1}$; at an axis for flow without swirl $y^{+} \sim|\delta t|^{-\frac{3}{2}}$. 


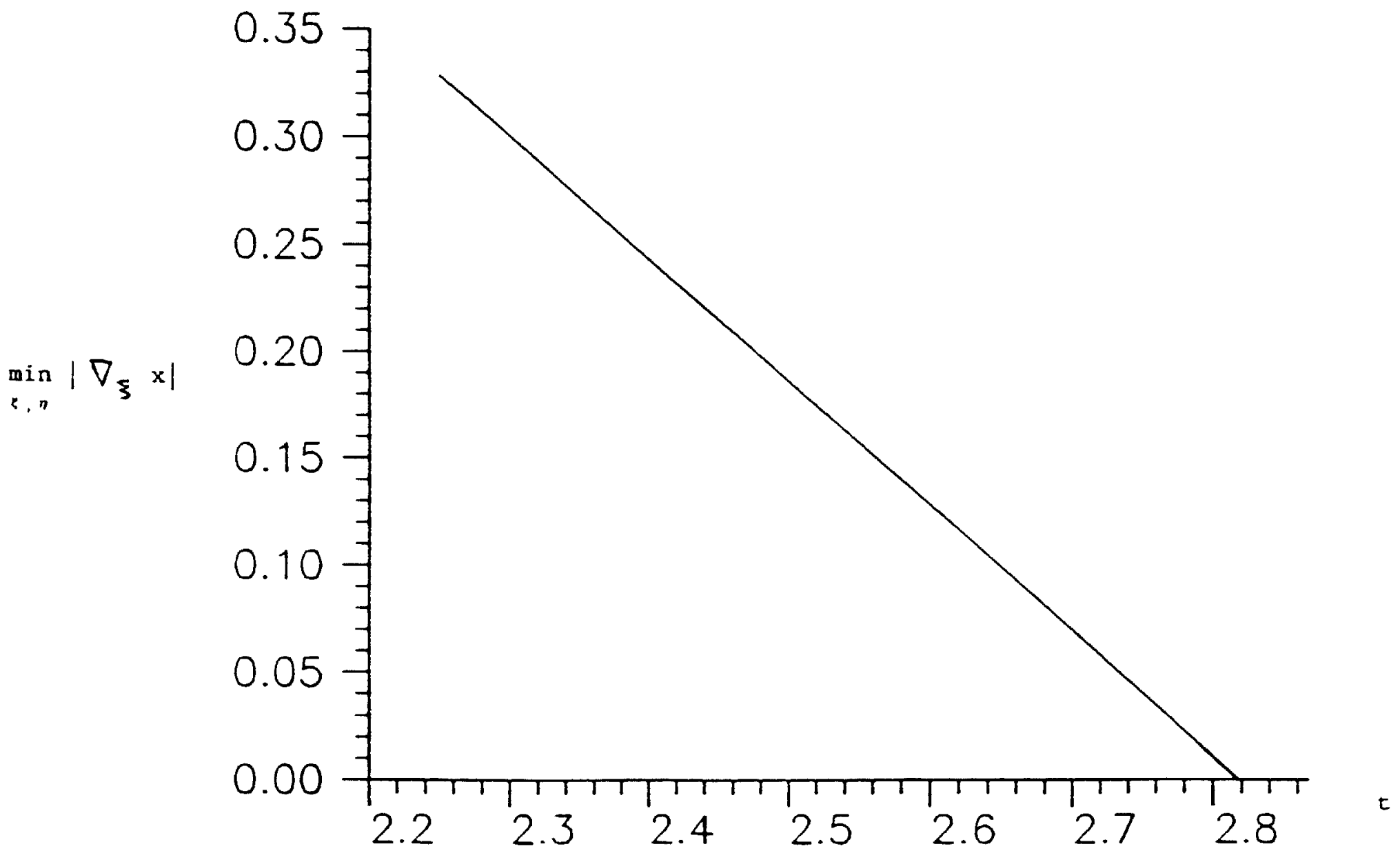

Figure 5. Boundary-layer collision in a curved pipe. (a) $\min _{\xi, \eta}\left|\nabla_{\xi} x\right|$ against time. 


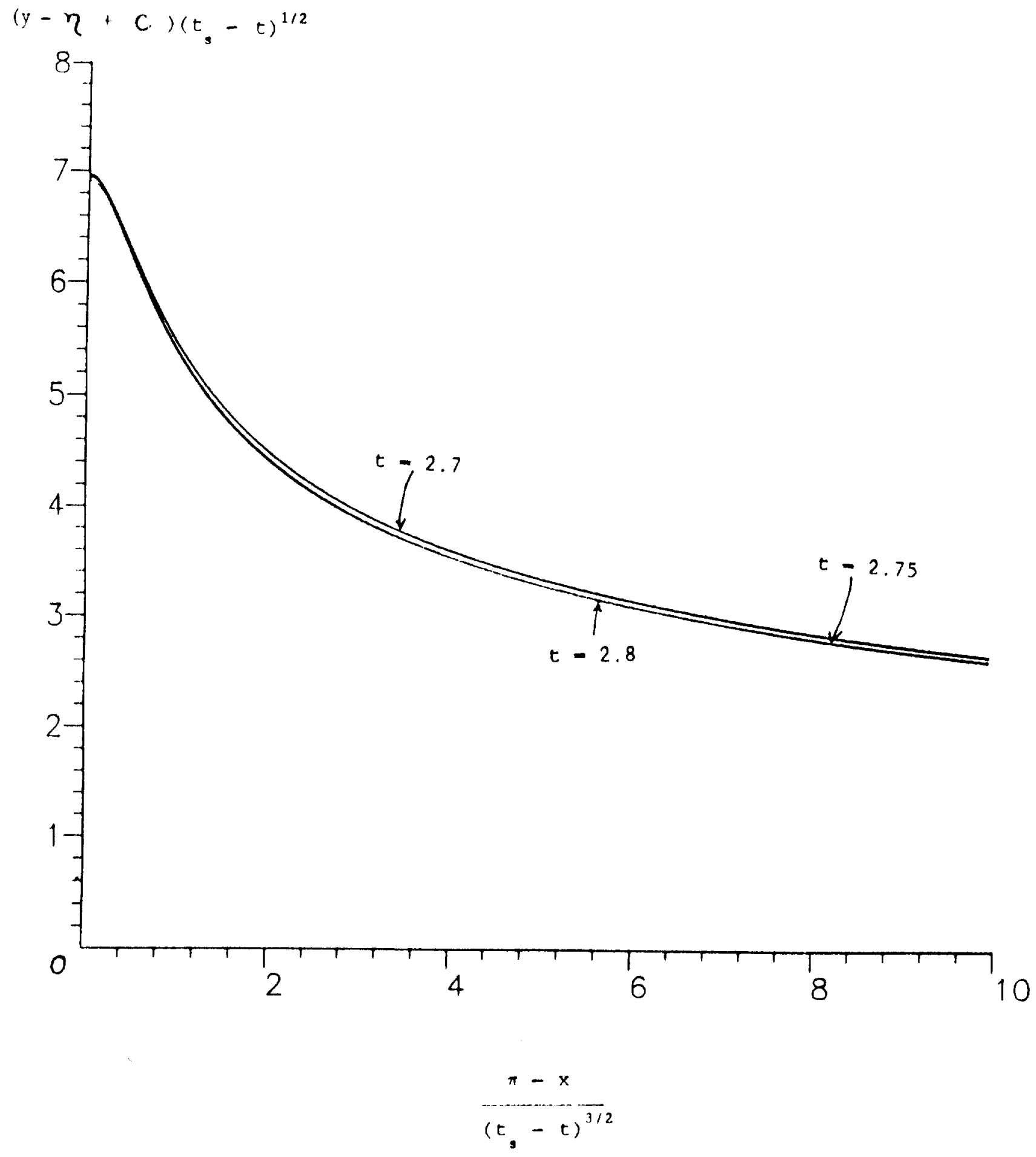

Figure 5. Boundary-layer collision in a curved pipe. (b) Scaled boundary-layer thickness for various times. 


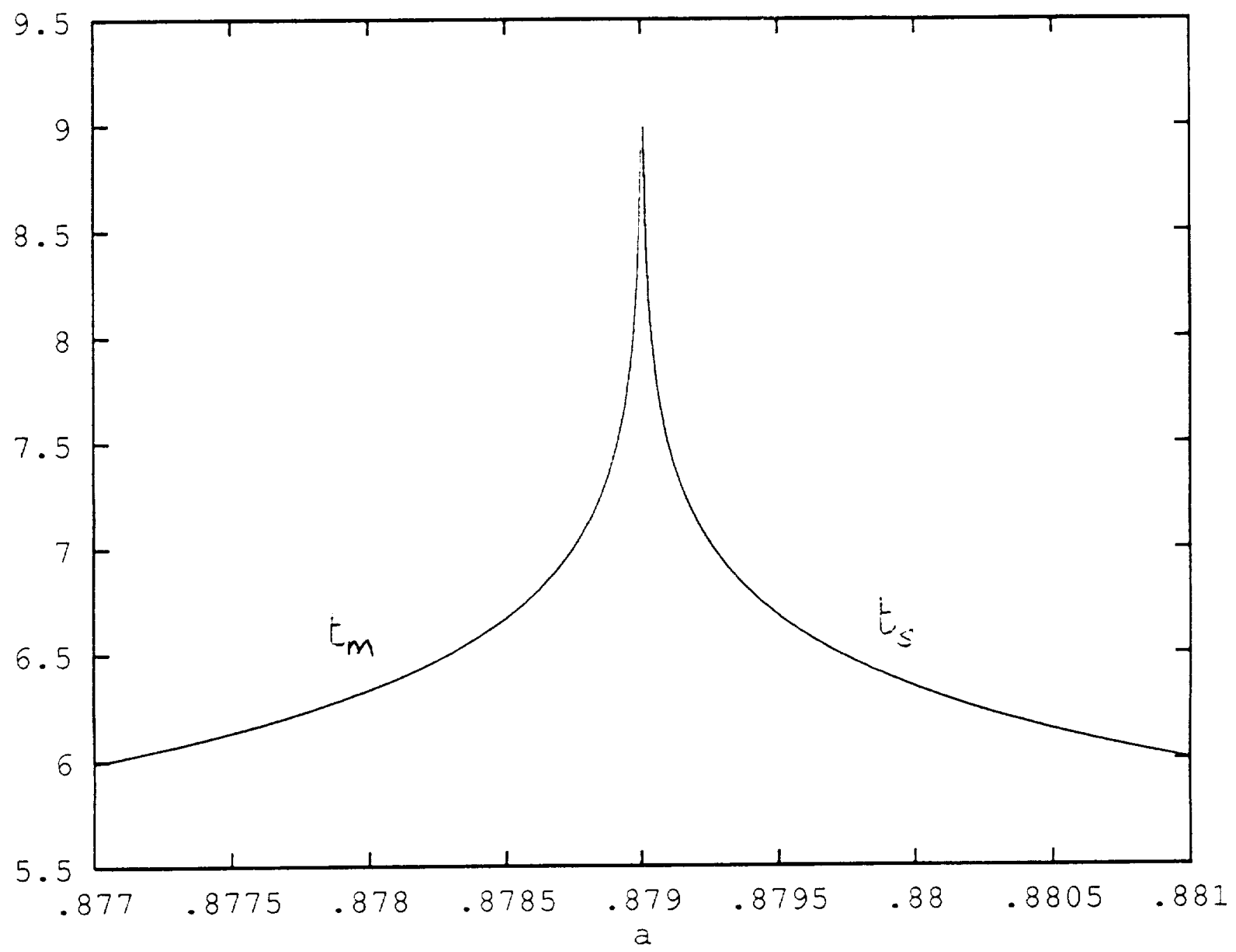

Figure 6. Properties of the numerical solution for the symmetry-line separation flow governed by $(5.5,6)$. (a) Plots of the separation time, $t_{s}\left(a>a_{s}\right)$, and the time, $t_{m}$, at which $x_{, \xi}$ attains its minimum value $\left(a<a_{0}\right)$. 


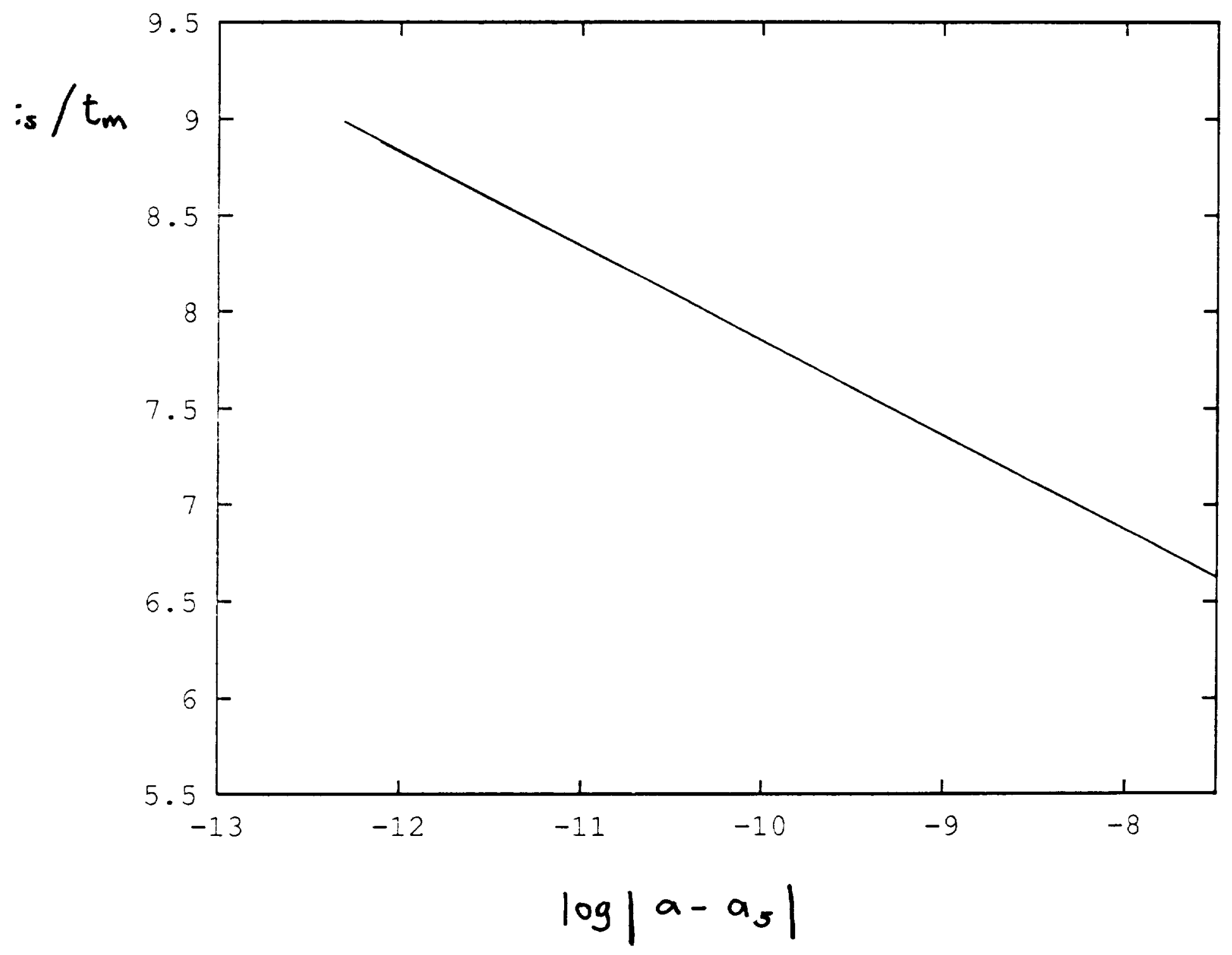

Figure 6. Properties of the numerical solution for the symmetry-line separation flow governed by $(5.5,6)$. (b) Plots of $t_{0}$ and $t_{m}$ against $\ln \left|a-a_{0}\right|$ (almost indistinguishable). 


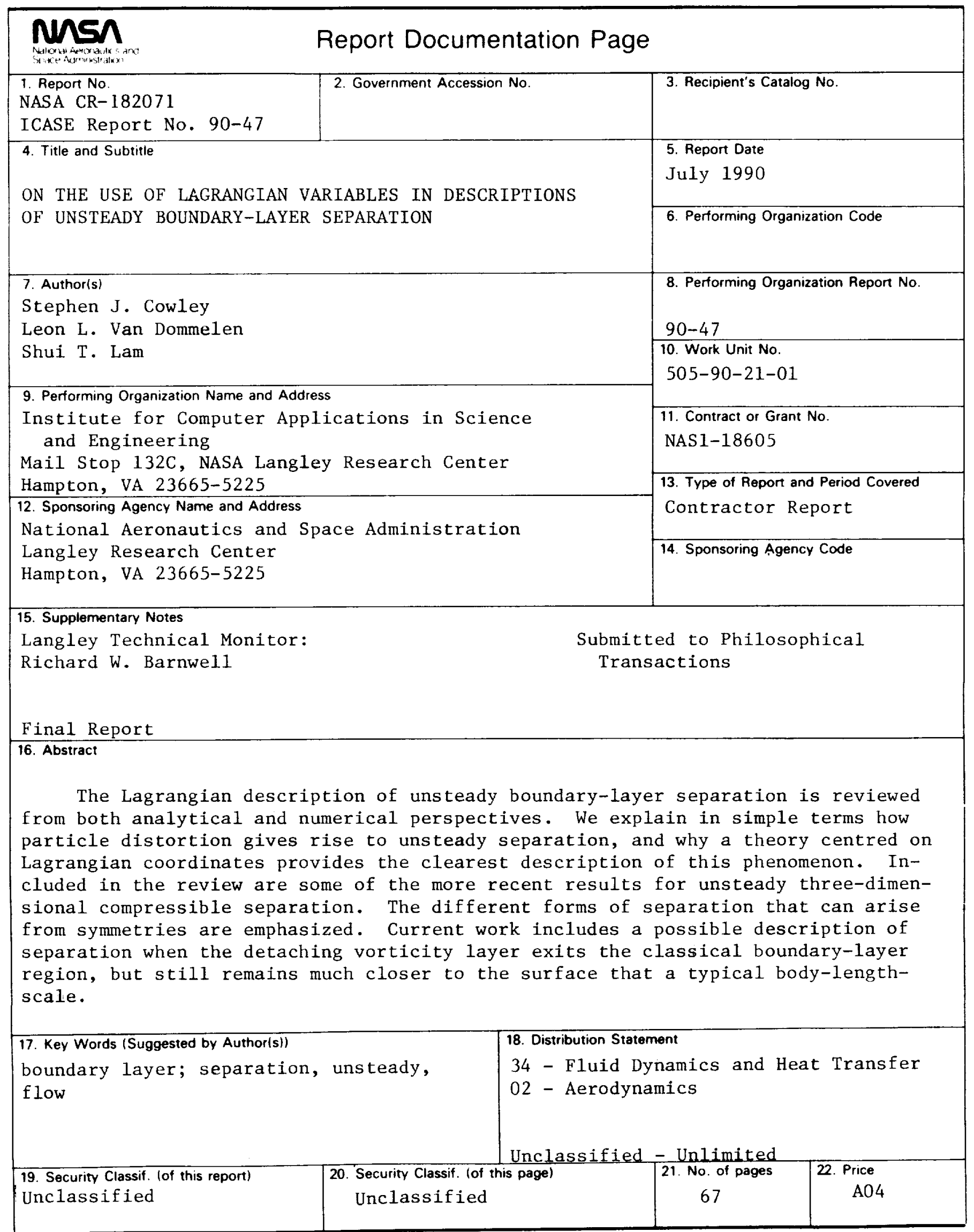

NASA FORM 1626 OCT 86 